\title{
Dynamics of thin current sheets: Cluster observations
}

\author{
W. Baumjohann ${ }^{1}$, A. Roux ${ }^{2}$, O. Le Contel ${ }^{2}$, R. Nakamura ${ }^{1}$, J. Birn ${ }^{3}$, M. Hoshino ${ }^{4}$, A. T. Y. Lui ${ }^{5}$, C. J. Owen ${ }^{6}$, \\ J.-A. Sauvaud ${ }^{7}$, A. Vaivads $^{8}$, D. Fontaine ${ }^{2}$, and A. Runov ${ }^{1}$ \\ ${ }^{1}$ Space Research Institute, Austrian Academy of Sciences, Graz, Austria \\ ${ }^{2}$ CETP-IPSL-CNRS, Vélizy, France \\ ${ }^{3}$ Los Alamos National Laboratory, Los Alamos, NM, USA \\ ${ }^{4}$ University of Tokyo, Tokyo, Japan \\ ${ }^{5}$ JHU/APL, Laurel, MD, USA \\ ${ }^{6}$ Mullard Space Science Laboratory, Univ. College London, UK \\ ${ }^{7}$ CESR/CNRS, Toulouse, France \\ ${ }^{8}$ Swedish Institute of Space Physics, Uppsala, Sweden
}

Received: 25 January 2007 - Accepted: 24 May 2007 - Published: 29 June 2007

\begin{abstract}
The paper tries to sort out the specific signatures of the Near Earth Neutral Line (NENL) and the Current Disruption $(\mathrm{CD})$ models, and looks for these signatures in Cluster data from two events. For both events transient magnetic signatures are observed, together with fast ion flows. In the simplest form of NENL scenario, with a large-scale two-dimensional reconnection site, quasi-invariance along $\mathrm{Y}$ is expected. Thus the magnetic signatures in the $\mathrm{S} / \mathrm{C}$ frame are interpreted as relative motions, along the $\mathrm{X}$ or $\mathrm{Z}$ direction, of a quasi-steady X-line, with respect to the S/C. In the simplest form of $\mathrm{CD}$ scenario an azimuthal modulation is expected. Hence the signatures in the $\mathrm{S} / \mathrm{C}$ frame are interpreted as signatures of azimuthally (along $\mathrm{Y}$ ) moving current system associated with low frequency fluctuations of $J_{y}$ and the corresponding field-aligned currents $\left(J_{x}\right)$. Event 1 covers a pseudo-breakup, developing only at high latitudes. First, a thin $\left(H \approx 2000 \mathrm{~km} \approx 2 \rho_{i}\right.$, with $\rho_{i}$ the ion gyroradius $)$ Current Sheet (CS) is found to be quiet. A slightly thinner CS $\left(H \approx 1000-2000 \mathrm{~km} \approx 1-2 \rho_{i}\right)$, crossed about 30 min later, is found to be active, with fast earthward ion flow bursts $(300-600 \mathrm{~km} / \mathrm{s})$ and simultaneous large amplitude fluctuations $(\delta B / B \sim 1)$. In the quiet $C S$ the current density $J_{y}$ is carried by ions. Conversely, in the active CS ions are moving eastward; the westward current is carried by electrons that move eastward, faster than ions. Similarly, the velocity of earthward flows $(300-600 \mathrm{~km} / \mathrm{s})$, observed during the active period, maximizes near or at the CS center. During the active phase of Event 1 no signature of the crossing of an X-line is identified, but an X-line located beyond Cluster could account for the observed ion flows, provided that it is active for at least $20 \mathrm{~min}$. Ion flow bursts can also be
\end{abstract}

Correspondence to: $\mathrm{W}$. Baumjohann

(baumjohann@oeaw.ac.at) due to $\mathrm{CD}$ and to the corresponding dipolarizations which are associated with changes in the current density. Yet their durations are shorter than the duration of the active period. While the overall $\partial B_{z} / \partial t$ is too weak to accelerate ions up to the observed velocities, short duration $\partial B_{z} / \partial t$ can produce the azimuthal electric field requested to account for the observed ion flow bursts. The corresponding large amplitude perturbations are shown to move eastward, which suggests that the reduction in the tail current could be achieved via a series of eastward traveling partial dipolarisations/CD. The second event is much more active than the first one. The observed flapping of the CS corresponds to an azimuthally propagating wave. A reversal in the proton flow velocity, from -1000 to $+1000 \mathrm{~km} / \mathrm{s}$, is measured by CODIF. The overall flow reversal, the associated change in the sign of $B_{z}$ and the relationship between $B_{x}$ and $B_{y}$ suggest that the spacecraft are moving with respect to an $\mathrm{X}$-line and its associated Hall-structure. Yet, a simple tailward retreat of a largescale X-line cannot account for all the observations, since several flow reversals are observed. These quasi-periodic flow reversals can also be associated with an azimuthal motion of the low frequency oscillations. Indeed, at the beginning of the interval $B_{y}$ varies rapidly along the $\mathrm{Y}$ direction; the magnetic signature is three-dimensional and essentially corresponds to a structure of filamentary field-aligned current, moving eastward at $\sim 200 \mathrm{~km} / \mathrm{s}$. The transverse size of the structure is $\sim 1000 \mathrm{~km}$. Similar structures are observed before and after. These filamentary structures are consistent with an eastward propagation of an azimuthal modulation associated with a current system $J_{y}, J_{x}$. During Event 1 , signatures of filamentary field-aligned current structures are also observed, in association with modulations of $J_{y}$. Hence, for both events the structure of the magnetic fields and currents is three-dimensional.

Published by Copernicus Publications on behalf of the European Geosciences Union. 
Keywords. Magnetospheric physics (Magnetotail; Plasma sheet) - Space plasma physics (Plasma waves and instabilities; Magnetic reconnection)

\section{Introduction}

Sudden releases of large amounts of magnetic energy, presumably due to plasma instabilities, occur during magnetospheric substorms. The plasma confinement is lost over a short time interval, while electrons and ions are accelerated, heated, and precipitated onto the upper atmosphere, which leads to the formation of auroras. Before this rapid heating/acceleration phase, the magnetic energy is slowly accumulated in the system, which leads to the formation of thin current sheets. Thus the quasi-steady formation of a thin current sheet seems to be a necessary step, whereby the conditions for an explosive release of magnetic energy are being set up. While this sequence of events is relatively well documented, thanks to numerous in-situ and remote sensing observations, there is no consensus yet about the process(es) that trigger(s) substorms. At the present time, two main scenarios are considered for magnetotail activity relevant for substorms. Large-scale MHD simulations of tail dynamics (Birn and Hesse, 1991, 1996; Hesse and Birn, 1991; Scholer and Otto, 1991; Birn et al., 1999) suggest that both plasmoid ejection and current reduction and diversion, described as the substorm current wedge (e.g. McPherron et al., 1973) are initiated by the formation of an X-line, causing both tailward and earthward plasma flow. The braking of the earthward flow in the inner tail leads to pile-up of magnetic flux and hence a dipolarization of the magnetic field (Hesse and Birn, 1991; Shiokawa et al., 1997; Baumjohann et al., 1999; Baumjohann, 2002) the diversion of the flow and the associated shear distort the magnetic field and build up the field-aligned currents of the substorm current wedge. This model is commonly referred to as the "near-Earth neutral line model" (Baker et al., 1996).

An alternative scenario, usually called "current disruption model," assumes that a substorm is triggered locally in the inner magnetotail, presumably by an instability that involves a cross-tail wave vector component (Lui, 1991). Potential candidates are cross-field current-driven instabilities (e.g. Lui, 1991) or interchange/ballooning modes (Roux et al., 1991; Hurricane et al., 1997; Pu et al., 1997; Bhattacharjee et al., 1998a,b; Cheng and Lui, 1998). The disruption of the perpendicular current can also be due to the interruption of the parallel current by an instability (Perraut et al., 2000). In the current disruption scenario, the formation of an X-line might be a later consequence of the dynamic evolution (Lui, 1996).

In order to resolve at least part of the "substorm controversy", theorists as well as data analysts, formed a "Substorm Onset Physics" team, that met twice at the International Space Science Institute in Bern, Switzerland. The team aimed at using data from the Cluster mission to discern between the two competing models. After some discussion, it was decided to focus on one of the key differences between the two models: the distinction between the waves that perturb the thin current sheet at substorm onset and their role in initiating the onset. The neutral line model is characterized by variations along the tail axis, whereas current disruption models are based on modes propagating azimuthally, i.e. parallel or antiparallel to the cross-tail current.

Hence, the team selected three periods when the Cluster spacecraft observed thin current sheet. Three intervals can give three examples only, but they were selected to cover a quiet thin current sheet, a thin current sheet during a weak substorm, and a thin current sheet during a storm-time substorm, and are thus thought to be representative for typical magnetospheric conditions.

\section{Models and signatures}

From the data which will be presented in Sects. 3 and 4 it will be clear that thin current sheets can be present in the magnetotail under a variety of conditions, ranging from relatively quiet through modestly active to very active, strongly driven scenarios. Accordingly we will discuss here relevant theories and modeling results including quasi-static models as well as instabilities related to thin current sheets.

In the following sections we will first present some major results from quasi-static models, then address details and implications of the near-Earth neutral line scenario and finally of the current disruption model(s). These models are not mutually exclusive. Rather, they may apply simultaneously, for instance, a relatively quiet, quasi-static, structure may exist within a propagating wave mode. Or they may be causally related; for instance, a small-scale wave mode may be necessary to provide the dissipation necessary for a large-scale mode, or the large-scale mode may lead to flows that become turbulent and thus drive smaller scale modes or modes with different wave vectors. A major distinction between the two substorm scenarios detailed in the Introduction is not whether current disruption occurs in one but not the other, but rather whether the responsible mode vectors are primarily in the $\mathrm{X}$ direction, along the tail, as in the simplest reconnection scenario, or in the y direction, across the tail, as in the simplest current disruption models. However, as indicated above, this distinction also may be an oversimplification. Further, as discussed below, negative $B_{z}$ values, generally thought to be the consequence of magnetic reconnection, may also arise as a large-amplitude nonlinear stage of a cross- tail mode. The breakdown of ideal MHD, frozen-in fields, and conservation of magnetic topology is a crucial feature in that case, as in reconnection. 


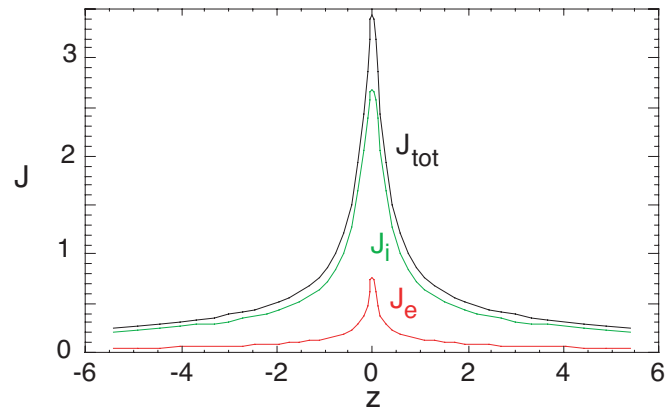

$\rho_{i}=0.01$
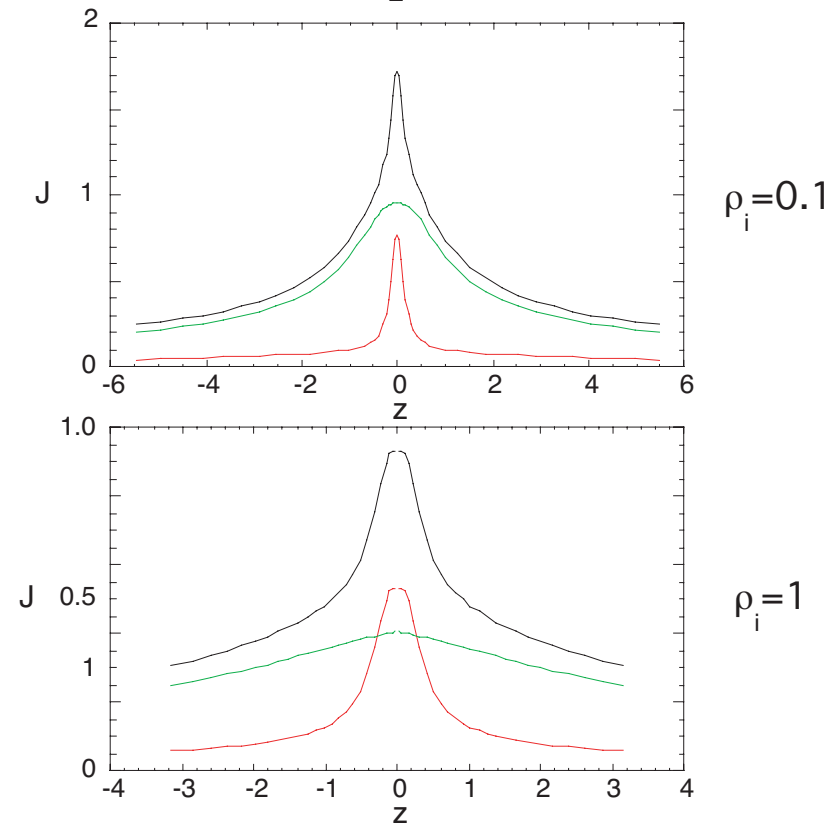

$\rho_{i}=1$

Fig. 1. Ion (green) and electron (red) contributions to the total current (black) in self-consistent models of a thin current sheet embedded in a wider current sheet, for various ion gyroradii scaled by the half-thickness of the wide current sheet; modified after Schindler and Birn (2002).

\subsection{Quasi-equilibrium models}

Quasi-static thin current sheet structures in the magnetotail may arise from the response to deformations imposed by the solar wind (Birn and Schindler, 2002). Both MHD and particle simulations consistently demonstrate that a thin current sheet can form as the consequence of the addition of magnetic flux to the lobes (Schindler and Birn, 1993; Pritchett and Coroniti, 1994; Hesse et al., 1996) An important role is played by the variation in $X$, the Earth-Sun direction (Birn et al., 1998). Quasi one-dimensional compression leads only to a moderate current density increase. However, a finite, not necessarily short-scale, variation in $X$ can produce a local current density enhancement that is much stronger. Particle and MHD simulations show qualitatively similar behavior, but kinetic effects modify the current sheet structure when the thickness approaches, or becomes less than, a typical ion

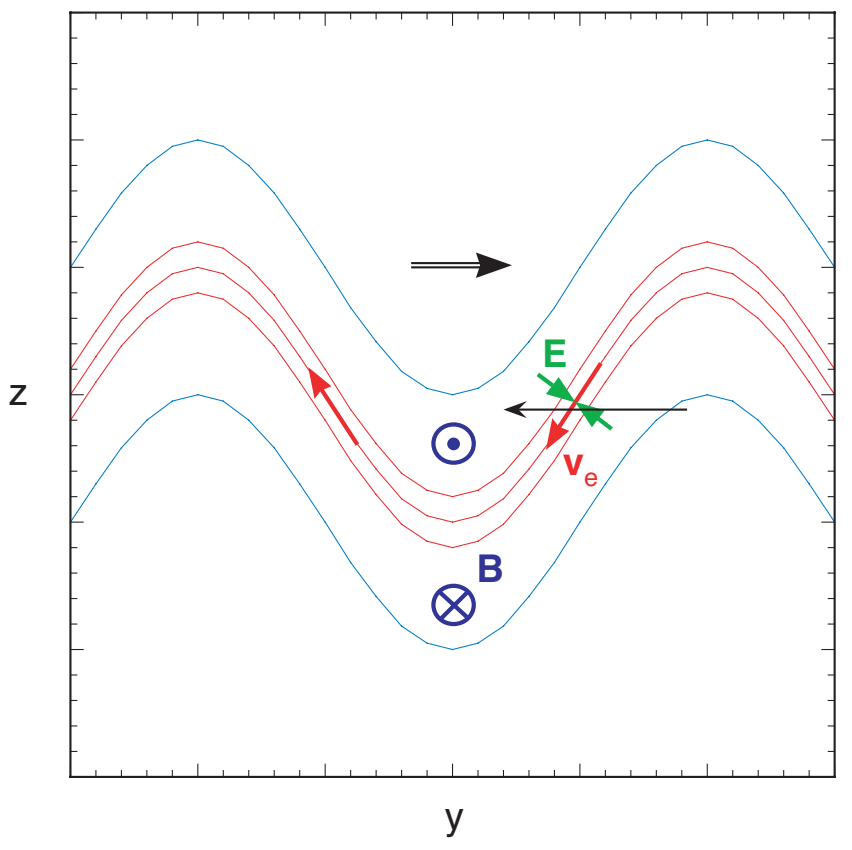

Fig. 2. Thin current sheet embedded in a wider current sheet which undergoes a kink mode propagating in $\mathrm{y}$, as indicated by the double arrow. The red arrow indicates the fast electron drift in the embedded current sheet. The green arrows indicate the electric field associated with that drift. The blue circles with a cross and a dot, respectively, show the magnetic field direction, and the single black arrow indicates the motion of the Cluster satellites relative to the moving structure.

gyroradius, as defined by the field strength outside the current sheet. Such thin sheets have actually been detected by Cluster (e.g., Nakamura et al., 2002, 2006a).

Recently, Schindler and Birn (2002) derived selfconsistent models of thin current sheets embedded within a wider plasma/current sheet. These models are solutions to the Vlasov equations of collisionless plasmas. They can hence serve to illustrate the changes in structure and the contributions to the currents under various scales. Two main results are of relevance here.

When the thickness of the current sheet becomes comparable to, or smaller than, a typical ion gyroradius or ion inertia length, the ion contribution to the thin current sheet is smeared out, so that the current in the thin sheet becomes dominated by the electrons. This is illustrated by Fig. 1, which shows the current contributions in thin embedded current sheets for 3 values of the ion gyroradius, scaled by the width of the wider current sheet.

Self-consistent equilibrium solutions generally require an electrostatic potential. For two-dimensional configurations with $E_{y}=0$, when the ion and electron distributions are functions of the total energy and the canonical momentum $P_{y}$, the potential is constant along field lines and corresponds to an electric field perpendicular to the current sheet (Schindler 

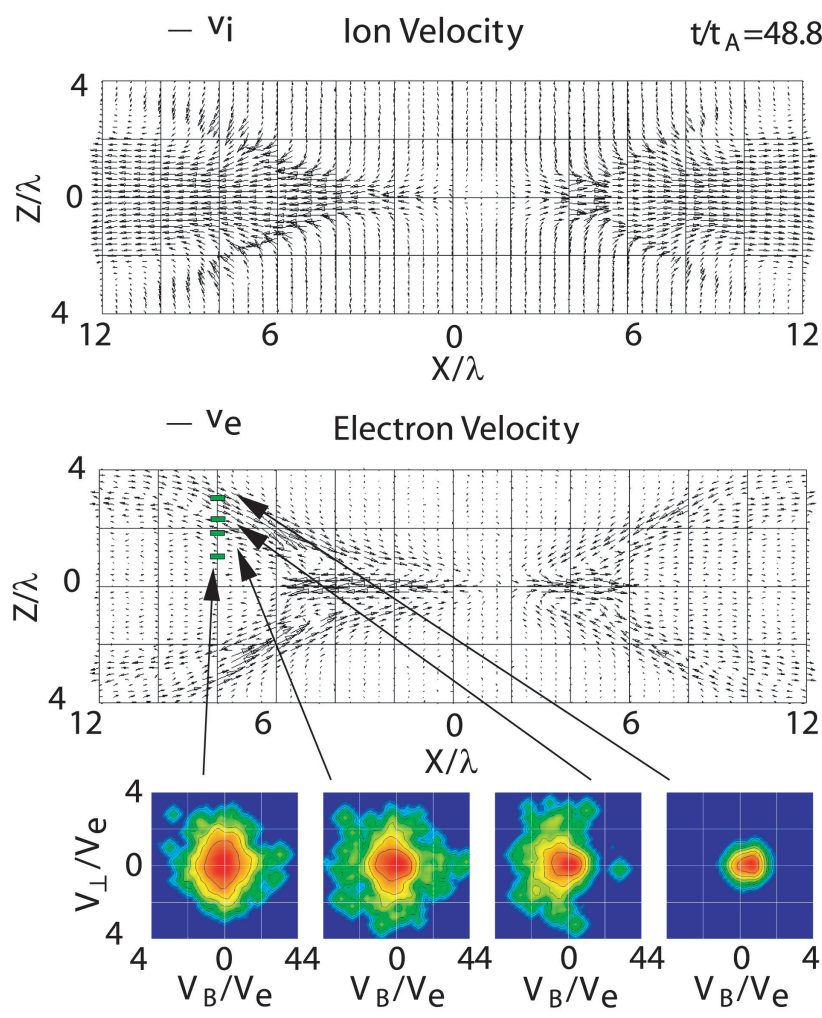

Fig. 3. Ion and electron flow velocity vectors in the $\mathrm{x}, \mathrm{z}$ plane obtained in a particle-in-cell simulation of collisionless magnetic reconnection by Hoshino et al. (2001). The lengths of velocity vectors are normalized by the initial ion and electron thermal speed, respectively. The bottom panels show electron velocity distributions obtained at the indicated locations.

and Birn, 2002). The value of the electrostatic potential, however, depends on the working frame. In the case of the Harris sheet the electrostatic potential is zero in a frame in which the electric field outside the current sheet vanishes, but in another frame a finite electrostatic potential is requested to ensure quasi-neutrality. The $\boldsymbol{E} \times \boldsymbol{B}$ drift of electrons in the electric field corresponding to a thin embedded current sheet (not shared by the ions for thin sheets) can carry the electric current associated with the thin sheet. In a locally planar, one-dimensional model, the electric field is directed towards the centre of the current sheet ( $\mathrm{Z}$ direction), while a tilt or a corrugation of the current sheet through kink modes, as recently observed by the Cluster and Geotail satellites (e.g. Zhang et al., 2002; Sergeev et al., 2003, 2004, 2006), would generate an additional y component of the electric field, as illustrated in Fig. 2.

\subsection{Magnetic tearing or reconnection, kinetic models}

The understanding of the physics of collisionless magnetic reconnection and of the (two-dimensional) field structure in the vicinity of the reconnection site has increased consider- ably over the years from a large number of simulations (e.g. Hewett et al., 1988; Pritchett, 1994, 2001; Tanaka, 1995a,b; Hesse et al., 1995, 1999, 2001a; Hesse and Winske, 1998). In the following we will discuss the current sheet structure expected from the magnetic reconnection model, based on particle-in-cell simulation results by Hoshino et al. (2001).

Figure 3 shows ion (top) and electron (bottom) velocity vectors in the $X Z$ plane, together with four electron distribution functions. The magnitudes of the ion and electron flow vectors are normalized by the initial ion and electron thermal velocity, respectively. The ion flows are basically directed from the X-type region to the O-type region, and the electron flow is also in the same direction in the plasma sheet. In the outflow region $(|X|>6)$, both ions and electrons have the same bulk velocity of $0.6 V_{t h i}$ or $\sim 0.5 V_{A}$, where $V_{t h i}$ and $V_{A}$ are the ion thermal and the Alfvén speeds, respectively. Inside that region the ions are unmagnetized and ion and electron flow speeds differ ("Hall region"). There is "cold" electron flow near the outer boundary between the lobe and the plasma sheet, directed toward the X-type region, and outward electron flow inside this boundary, which consists of two populations of "cold" electrons and "hot" electron beams. These flows are associated with Hall electric currents in a thin plasma sheet with a thickness of the order of the ion inertia length.

Inside the ion diffusion region near the $\mathrm{X}$-type point where the ions are unmagnetized, the electron flow becomes faster than the ion flow. The magnetized electrons can have a large $\boldsymbol{E} \times \boldsymbol{B}$ drift velocity in a weak magnetic field region, which can become larger than the Alfvén velocity. This relative flow difference between ions and electrons produces Hall currents in the reconnection plane, corresponding to quadrupolar dawn- dusk magnetic fields as shown in the second panel of Fig. 4. Maintaining the continuity of the electric current, a field-aligned electric current is generated in the outer boundary, associated with field-aligned electron motion. The dawn-dusk electric field $E_{y}$ is positive in the reconnection region, and the strongest electric field $E_{y}$ is found between the $\mathrm{X}$-type region and the O-type region. In addition to the global reconnection electric field, a small scale, bursty electric field structure can also be found. In the bottom panel, the amplitude of the electric fields in the reconnection plane, $\left|E_{x, z}\right|=\sqrt{E_{x}^{2}+E_{z}^{2}}$, is depicted. The strongest intensity can be found in the quadrupolar $B_{y}$ region (i.e. the Hall electric current region), whose thickness is of the order of ion inertia length. The polarization electric field toward the neutral sheet is produced by the inertia difference between ions and electrons across the boundary. In addition to the large-scale X-type structure of $E_{x, z}$, a small-scale structure embedded in the Hall current region can also be observed in the highspatial resolution simulation. This small-scale electric field may result from instabilities generated by the strong Hall current in the boundary region, such as the electron bump-on-tail and the Buneman instabilities. 
While the two-dimensional structure of the reconnection site appears well understood, the structure in the crosstail direction is less well explored. Due to the complexity of the problem and the numerical effort involved, only a few three-dimensional simulations of collisionless reconnection have been performed (e.g., Tanaka, 1995a; Pritchett and Coroniti, 1996). As a result, the question of whether the results from 2.5-dimensional models carry over to more realistic, three-dimensional configurations remains largely open. Recent three-dimensional simulations (Rogers et al., 2000; Hesse et al., 2001b; Zeiler et al., 2002) support the view that collisionless magnetic reconnection in 3-D current sheets operates in a manner very similar to the results derived from translationally invariant models. The development of structure in the out-of-plane direction appears to be limited to small scales which may enhance local dissipation coefficients (Huba et al., 1978; Büchner, 1998; Büchner and Kuska, 1999) but which do not alter the large-scale flow patterns.

\subsection{Cross-tail modes and current disruption}

Several cross-tail current instabilities are being considered for the generation of modes propagating across the tail: the lower-hybrid drift instability, the drift-kink instability, the drift-sausage instability, ballooning modes, KelvinHelmholtz modes, and the cross-field current instability. At short wavelengths, the lower hybrid drift instability (LHDI Huba et al., 1978) is strongly localized at the edge of the current sheet (Brackbill et al., 1984). Nonlinear development of the LHDI, however, can modify the initial equilibrium and/or drive secondary modes, discussed below. Horiuchi and Sato (1999) have proposed that the LHDI causes a thinning of the current sheet (not to be confused with the driven thinning caused by flux transfer from the dayside to the tail), as confirmed by simulations of Lapenta and Brackbill (2000). Another possibility, suggested by Hesse and Kivelson (1998), is that the LHDI generates a velocity shear on the edge of the current sheet, which in turn drives the Kelvin-Helmholtz instability, discussed below.

The drift-kink instability (DKI Zhu and Winglee, 1996; Pritchett and Coroniti, 1996; Pritchett et al., 1996; Ozaki et al., 1996; Lapenta and Brackbill, 1997; Nishikawa, 1997) grows at small and modest mass ratios $m_{i} / m_{e}$. However, linear theory, based on the numerical integration of the particle orbits (Daughton, 1999a,b), and kinetic simulations where the LHDI is suppressed (Hesse and Birn, 2000) predict very small growth rates at realistic mass ratio $m_{i} / m_{e}$. Nevertheless, a number of simulations see rapid kinking (Ozaki et al., 1996; Lapenta and Brackbill, 1997; Horiuchi and Sato, 1999), possibly driven by the nonlinear evolution of the LHDI. The drift-sausage (DS) mode has properties similar to the DK mode but with opposite parity. This mode has been observed in some simulations (Büchner, 1998; Büchner
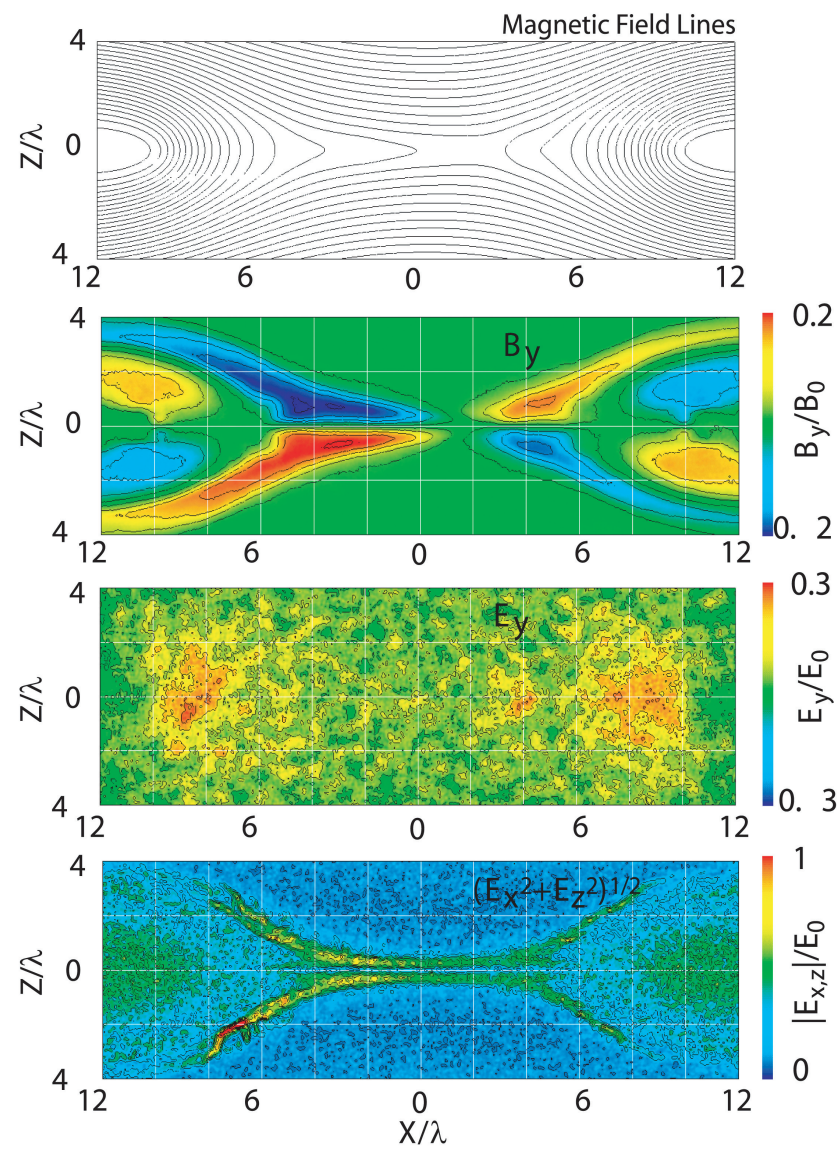

Fig. 4. Characteristic field structures obtained in a particle-in-cell simulation of collisionless magnetic reconnection (Hoshino, 2001). The magnetic field By is normalized by the initial lobe magnetic field $B_{0}$, and the electric field is normalized by $E_{0}=B_{0} V_{A} / c$. Note that the Earth is to the right in this figure.

and Kuska, 1999) and in approximate linear theories (see e.g. Yoon and Lui, 2001, for a review).

The Kelvin-Helmholtz $(\mathrm{KH})$ instability is a relatively long-wavelength, long-period mode and hence an attractive candidate for the observed $\sim 2$-min oscillations propagating across the tail. In an idealized configuration, where the magnetic field has no cross-tail component and the current, and hence the ion drift are in the cross-tail direction, there is no instability threshold, although the growth rate depends on the characteristic scale of the velocity shear. While a strict Harris equilibrium corresponds to uniform ion drift speed, and hence does not excite $\mathrm{KH}$ oscillations, there are several ways how the velocity profile might be modified and thus become susceptible to KH instability (e.g. Yoon et al., 1996). As mentioned above, small-scale fluctuations, such as LHD modes, can modify the current distribution and hence the velocity profile (e.g. Hesse and Kivelson, 1998; Daughton, 2002). Also, as illustrated in Fig. 1, when a thin current sheet is embedded in a wider one, as often observed by Cluster 


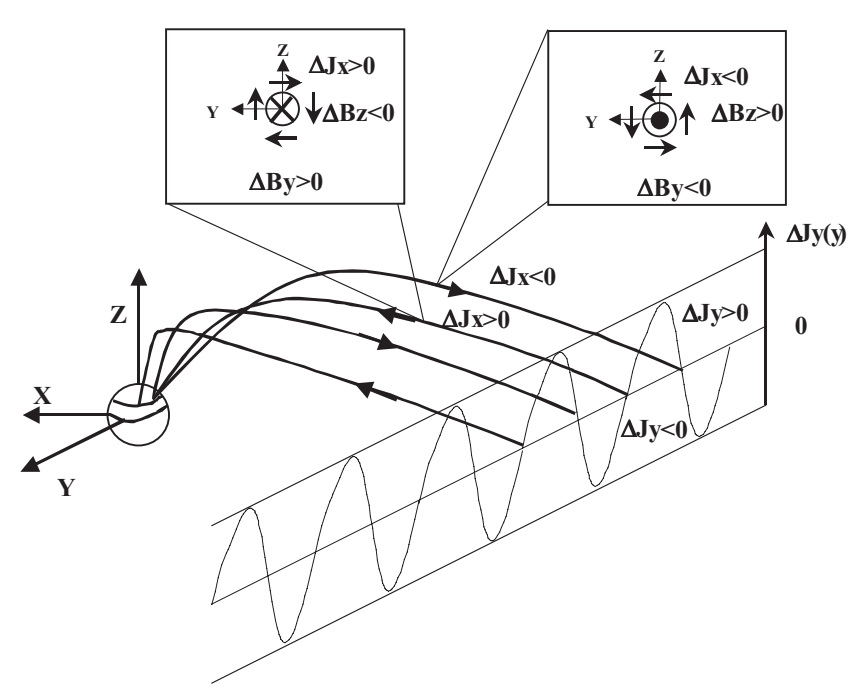

Fig. 5. Schematic view of the parallel current system corresponding to an azimuthal modulation of the cross tail current $J_{y}$. The top panel inserts display the local magnetic field perturbation produced by a tailward (resp. earthward) parallel current $\left(\sim J_{x}\right)$.

(e.g. Asano et al., 2005; Runov et al., 2006), the thin one tends to be carried by the electrons, while the wider one is still carried by ions. In that case, the ion speed in the center would be reduced (and could even be reversed). A KH analysis by Lapenta and Knoll (2003) indeed shows that KH instability can be excited and propagate in the general current and ion flow direction, even when the ion flow velocity is reversed in the center.

These modes may provide an explanation for waves propagating across the tail, but they do not necessarily lead to a net reduction and diversion of the cross-tail current. There are two major plasma instabilities considered for such current disruption. The first one is the current-driven electromagnetic ion cyclotron instability (Perraut et al., 2000). For this model, the formation of a thin current sheet during the growth phase is described as an externally applied time-dependent perturbation, localized in the azimuthal direction. The timescale of the perturbation is assumed to be larger than the ion and electron bounce periods. The kinetic response of the plasma, taking into account the bounce motion of particles due to the mirror geometry of the near-Earth magnetotail, implies the development of an electrostatic potential constant along a given magnetic field line (Hurricane et al., 1995). The corresponding potential electric field tends to shield the induced electric field due to the stretching of the magnetic field lines. Therefore the perpendicular motion, at least in the near equatorial region, is partly inhibited (Le Contel et al., 2000a,b). This can explain why CS thinning (or oscillations of CS) are not necessarily accompanied by an azimuthal $E_{y}$, and hence by an earthward or tailward flow.

On the other hand, the increase of the cross-tail current in an azimuthally localized region, during the growth phase implies an increase of the parallel current in order to ensure the zero divergence of the total current $\left(\nabla \cdot \mathbf{j} \sim \partial j_{y} / \partial y+B \partial / \partial l\left(j_{\|} / B\right) \sim 0\right.$, where $l$ is the length along a field line). The radial component of the current can be neglected assuming that the radial scale length of the perturbation is larger than the azimuthal and field-aligned scale lengths. For a large enough parallel current, "highfrequency" current- driven Alfvén waves (CDA) in the range of proton cyclotron frequencies are driven unstable. As they propagate along field lines, CDA waves can undergo two types of resonances. In the CS the waves interact with electron via bounce resonance. As they propagate away from the equatorial region, CDA waves are mode converted into shear Alfvén (SA) waves and the phase velocity (essentially the Alfvén speed) becomes of the order of the electron thermal velocity. In such conditions, CDA/SA waves are able to produce electron parallel diffusion. For intense CDA/SA waves, the diffusion time $\left(\tau_{d}\right)$ of electrons via CDA/SA waves is equivalent to the bounce time $\left(\tau_{b}\right)$, which has two important consequences (Le Contel et al., 2001b,a): (1) The parallel current is disrupted, therefore the equilibrium is broken and the perpendicular current must also vanish, thereby producing a local dipolarization, in agreement with observations; and (2) the non-local response associated with the electron bounce motion vanishes. The induced electric field corresponding to the local dipolarization, is no longer shielded and produces transient fast flows. Therefore, on the timescale of electron diffusion, large electric fields can exist and produce enhanced electric drift and the corresponding fast flows.

The previous scenario has been described in the context of the quasi-static evolution of a current sheet, for instance during the growth phase. It also applies to a situation where low-frequency modes, for instance ballooning modes, spatially modulate the $J_{y}$ current (Pellat et al., 2000). As depicted in Fig. 5, the spatial modulation of $J_{y}$ implies a series of field aligned currents that eventually turn out to be unstable, when the parallel current increases, that is when the parallel drift between electrons and ions gets large enough. The magnetic signatures of these parallel currents are also depicted in Fig. 5. At Cluster orbit, parallel currents will essentially be radial, hence the corresponding magnetic signatures, $\delta B$, will be in the $Y Z$ plane. Thus, in this model, the fluctuations observed in $B_{y}$ and $B_{z}$ are interpreted as signatures of parallel currents associated with azimuthally propagating structures carrying parallel currents (as illustrated in the figure). Thus the interpretation of the quadrupolar signature on $B_{y}$, and bi- polar signature on $B_{z}$, as the signature of Hall currents associated with an X-line (see Fig. 4) moving vertically (reversal of $B_{y}$ ) or radially (reversal of $B_{z}$ ), is not the only possibility for interpreting the data. This type of signature can also be produced by the 3-D system of current associated with low-frequency azimuthally propagating waves. Close to the equator, where the perturbations of the perpendicular current are localized (and where parallel currents vanish by symmetry), a negative $B_{z}$ can be obtained as 
soon as the absolute value of magnetic perturbation, associated with the perpendicular current perturbation, exceeds the weak dipole field. Off equator, where parallel current perturbations are stronger, correlated $B_{y}$ and $B_{z}$ perturbations can be associated with an azimuthal motion of the mode.

The second candidate for current driven instability is the cross-field current instability (Lui, 1991). For this paradigm, enhanced current density in the tail current sheet due to kinetic ballooning instability (Cheng and Lui, 1998) or to any process responsible for the explosive growth phase (Ohtani et al., 1992) is assumed to occur just prior to current disruption. This leads to the excitation of the cross-field current instability with high frequency perturbations from oblique whistler waves. The resultant development of this instability leads to turbulent environment with waves over a broad frequency range from about the ion cyclotron frequency to lower hybrid frequency. These oblique whistler waves can give rise to quadruple $B_{y}$ perturbations outside the current disruption region. This activity associated with this kinetic instability is spatially localized initially.

Most researchers associate moderate- and high-speed plasma flows with magnetic reconnection. However, current disruption can lead to force imbalance and consequent plasma acceleration to high-speed plasma flows as well (Lui et al., 1993). Figure 6 illustrates schematically the expected loss of equilibrium from current disruption (Lui, 2001). The top part of the figure shows that before occurrence of current disruption, the current density in the current sheet varies rather smoothly across the sheet. During current disruption, the current density becomes highly inhomogeneous, ranging from reversed current flow to enhanced current density with the overall current reduction averaged over the entire region. The nonlinear evolution of the plasma instability or instabilities responsible for current disruption leads to large magnetic fluctuations, especially in the $B_{z}$ component, as depicted in the middle of Fig. 6. In the near-Earth region, the ambient magnetic field component $B_{z}$ is strong. The net $B_{z}$ is thus mostly northward because the $B_{z}$ fluctuation seldom goes southward large enough to overcome the ambient field. In this region, the amount of current reduction due to disruption typically is smaller than the increase of magnetic field due to current reduction. This is because the current enhancement prior to current disruption suppresses the dipolar field contribution to the local magnetic field. Current reduction results in diminishing this suppression and thus an increase of magnetic field. There is thus a net increase in the $\mathbf{j} \times \mathbf{B}$ force in this situation. On the other hand, in the mid tail region the ambient magnetic field is weak. The net magnetic field therefore becomes frequently negative. When the net $B_{z}$ is negative, both $\mathbf{j} \times \mathbf{B}$ and pressure gradient forces accelerate the plasma tailward. Even when the net $B_{z}$ is positive, the current reduction can become larger than the associated magnetic field increase. This leads to a net decrease in the $\mathbf{j} \times \mathbf{B}$ force and, again, a consequent tailward plasma acceleration. The above consideration indicates that there would be

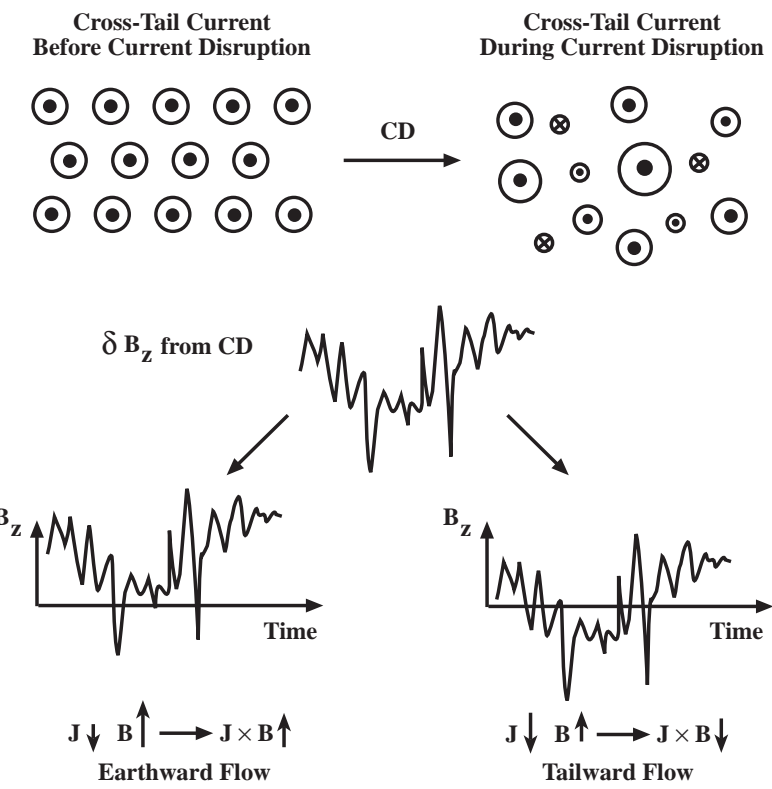

Fig. 6. Schematic of how small-scale fluctuations from localized current disruptions might lead to organized earthward or tailward flow. After Lui (2001).

some association between tailward plasma flow and southward $B_{z}$ but deviations from this association is expected to occur occasionally, e.g., tailward flow with northward $B_{z}$ or earthward flow with southward $B_{z}$.

\section{Description of events}

Thin current sheets with a thickness comparable or less than the Cluster tetrahedron scale are observed under different conditions. Here we describe current sheet crossings from two Cluster 2001 tail periods, when the tetrahedron scale was about $2000 \mathrm{~km}$ and the spacecraft were near apogee in the premidnight sector (Fig. 7). The first event, between 20:40 and 22:00 UT on 7 September 2001, consists of two different types of current sheet: (a) a thin current sheet during a quiet interval and (b) a current sheet during a pseudo-breakup. The second event shows (c) a current sheet with a flow reversal and hence a possible $\mathrm{X}$-line signature during a storm-time substorm, between 09:20 and 09:55 UT, on 1 October 2001. In the following we briefly describe the global context of the events in Sect. 3.1 and highlight the specific observed features for these events in Sects. 3.2-3.3. Finally, the key observations are summarized in Sect. 3.5.

\subsection{Overview of the selected events}

\subsubsection{September 2001}

The 7 September event occurred just before a change from a northward to a southward solar wind $B_{z}$ component, which 

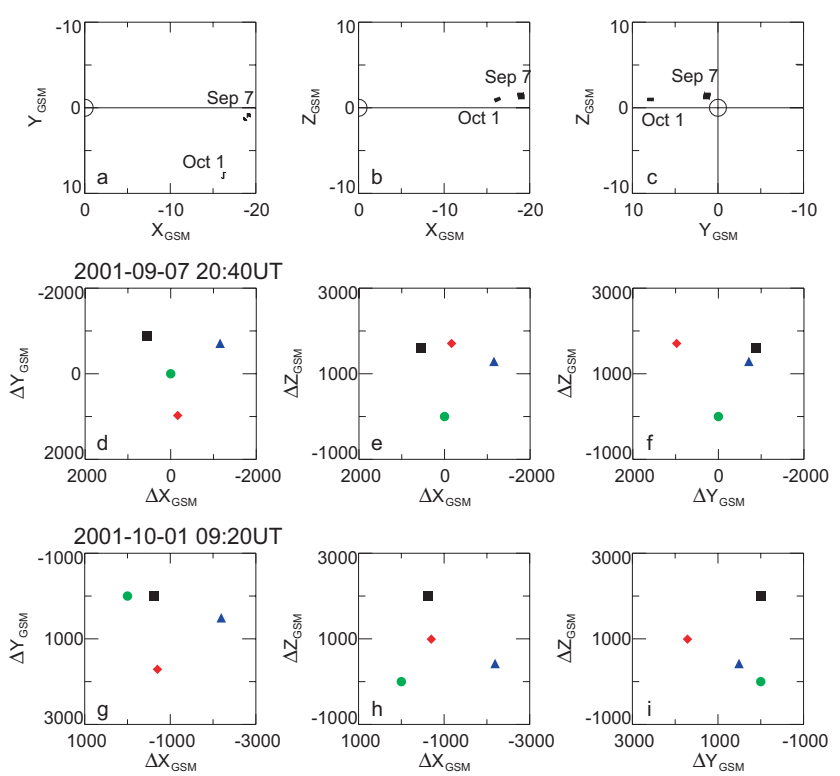

Fig. 7. Cluster location for the 7 September and 1 October events and tetrahedron configuration for 7 September and 1 October event.

happened at the end of the selected time interval, around 22:00 UT. The Image and Polar spacecraft (data not shown) give evidence for an auroral bulge developing near the Cluster footprint, at about 21:29 UT. This bulge has a small extension in latitude, around $70^{\circ}$ MLat. Hence Event 1 is not a fully developed substorm; it corresponds to a localized (in latitude) perturbation propagating eastward at $\sim 50 \mathrm{~km} / \mathrm{s}$ in the ionosphere, presumably a pseudo-breakup.

Figure 8 shows spin averaged field and particle data. Three components of the magnetic field in GSM coordinates obtained by the FGM magnetometer (Balogh et al., 2001) are shown in Figs. 8a-c. The DS2 component of the electric field data from EFW (Gustafsson et al., 2001) is shown in Fig. $8 \mathrm{~d}$ (the DS2 component is approximately parallel to $-\mathrm{Y}$ in GSE coordinates). Here we changed the sign of the DS2 component so that it is close to the dawn-to-dusk electric field, $E_{y} . \quad X$ and $Y$ components of the proton bulk flow from the CIS/CODIF experiment (Rème et al., 2001) are shown in Figs. 8e and f. $X$ and $Y$ components of the current density calculated from the linear curl estimator technique (Chanteur, 1998), using FGM data are shown in Fig. 8g. It should be kept in mind that Cluster estimates the averaged current density on the scale of the tetrahedron, i.e., $2000 \mathrm{~km}$. Finally, panels (h) and (i) show the parameter for protons and oxygen, respectively. Values of $\beta$ up to 100 are measured for protons and $\beta>1$, for oxygen. Yet, in spite of this high- $\beta$, thin CS remains stable and quiet.

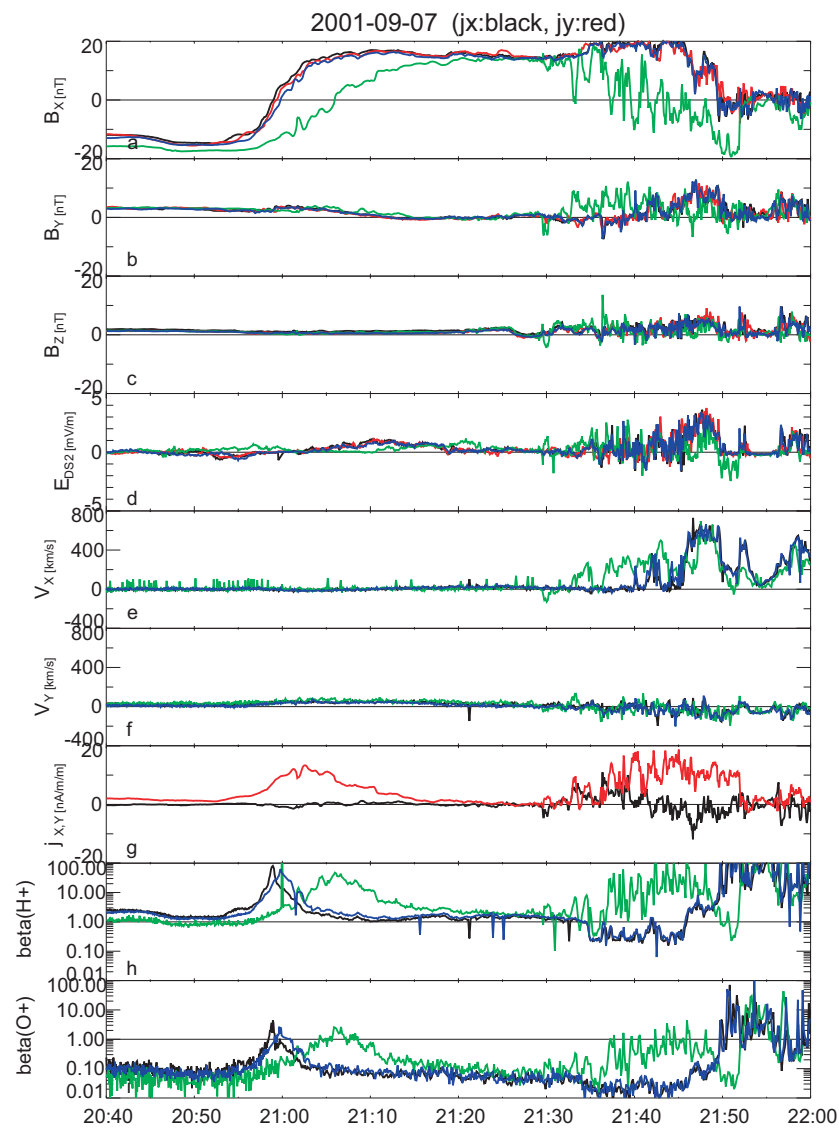

Fig. 8. (a, b, c): spin resolution data from GSM components of the magnetic field, (d) DS2 component of the electric field, (e, f) GSM X,Y components of the proton bulk velocity, (g) current density determined from the magnetic field, $(\mathbf{h}, \mathbf{i})$ proton and oxygen beta. For the particle and field plots, profiles for Cluster 1, 2, 3, 4 are plotted with black, red, green, and blue lines, respectively. Black and red lines in the current density plots correspond to $\mathrm{X}$ and Y components.

\section{Quiet CS crossing}

Between 20:40 and 21:30 UT, the $B_{x}$ components vary from approximately $-12 \mathrm{nT}$ (at S/C 3) to $+18 \mathrm{nT}$ (on S/C 4; see Fig. 8a), hence the four S/C cross the magnetic equator. During this crossing the CS is relatively quiet; the dawn-dusk electric field (Fig. 8d) and the ion flows in the $\mathrm{X}$ direction (Fig. 8e) are weak and the magnetic fluctuations are small (panels $\mathrm{a}, \mathrm{b}$, and $\mathrm{c}$ ). The most rapid variation is along the $\mathrm{Z}$ direction. Then the CS thickness can be estimated from the difference between the values of $B_{x}$, measured at the four S/C locations; this is done in Sect. 3.2.2. Around 21:00 UT $\mathrm{S} / \mathrm{C} 3$, which is at a lower $Z$ than its 3 companions, is located at the CS boundary, while S/C 1, 2 and 4 are located close to the magnetic equator. Hence the half-thickness of the current sheet should be of the order of the distance, projected along $Z$, between S/C 3 and the others: $\Delta Z_{\mathrm{GSM}}(3,4) \approx 1300 \mathrm{~km}$, 
at about 21:00 UT. Knowing the CS thickness, one can estimate the current density, $J_{y} \approx \Delta B_{x} / \mu_{0} H \approx 10 \mathrm{nA} / \mathrm{m}^{2}$, consistent with the value calculated from curl $\mathbf{B}$ (panel g). For this event, however, the characteristic spatial scale of the current sheet is comparable to the distance between the satellites. Therefore, the current density obtained from the curlometer method can only be considered as a rough estimate (in fact an underestimate). During this quiet CS crossing the ion flow velocity is sufficient to account for the estimated $J_{y}$. Indeed for $N \approx 1 / \mathrm{cm}^{3}$, and $V_{y} \approx 50 \mathrm{~km} / \mathrm{s}$ (estimated from CIS) we find $J_{y} \approx 8 \mathrm{nA} / \mathrm{m}^{2}$. Hence during this quiet CS crossing the current, in the $\mathrm{S} / \mathrm{C}$ frame, is essentially carried by ions.

\section{Active CS crossings}

From 21:30 to 21:42 UT, the averaged value of $B_{x}$, for S/C 3, varies from positive to negative. Large amplitude oscillations in $B_{y}$ and $B_{z}$ are superimposed. Hence, the current sheet structure is three-dimensional. On the other hand, the fluctuations in $B_{x}$, detected by S/C 1,2 and 4, are weaker, and $B_{x}$ remains close to the lobe value $(\sim 20 \mathrm{nT})$. Therefore S/C 1,2 and 4 are located outside the CS, or close to CS boundary layer, at least till 21:46 UT. Thus, between 21:30 and 21:46 UT, the CS thickness must be smaller than the distance between SC 3 and its companions projected along $Z$ $(\sim 1500 \mathrm{~km})$. After 21:45 UT $B_{x}$ at S/C 1, 2, 4 decrease, on average, and oscillate, while $B_{x}$ at S/C 3 becomes negative. Hence the CS thickness becomes comparable to the distance between the S/C. Using the value of $B_{x}$, normalized by the lobe value $(\sim 20 \mathrm{nT})$, as a proxy to estimate how far a given $\mathrm{S} / \mathrm{C}$ is located away from the center of the CS, we find that large ion flow velocities $\left(V_{x}\right)$ are found close to the equator. The reverse is not true: being at the equator does not warrant the observation of a fast ion flow. Notice that large amplitude variations in $E_{y}$ (Fig. 8d) are also observed.

Surprisingly the ion velocity in the $\mathrm{Y}$ direction, which was positive (as expected) before 21:30 UT, becomes negative (eastward) around 21:35 UT, and sometimes reaches $-200 \mathrm{~km} / \mathrm{s}$. The westward current must be carried by electrons moving eastward, faster than ions; this is further discussed in Sect. 3.2.2. Asano et al. (2004) have also given evidence for westward currents carried by electrons, in the case of a Geotail event.

\section{Thick CS}

After 21:52 UT, all s/c measure almost the same $B_{x} \sim 0$ indicating that the spatial scale of the CS is now much larger than the distance between the satellites. During this period, the current density $J_{y}$ (panel g) is smaller than before. Note that after 21:55 UT, a short lasting thinning of the CS occurs again, while enhanced $V_{x}$ is detected.

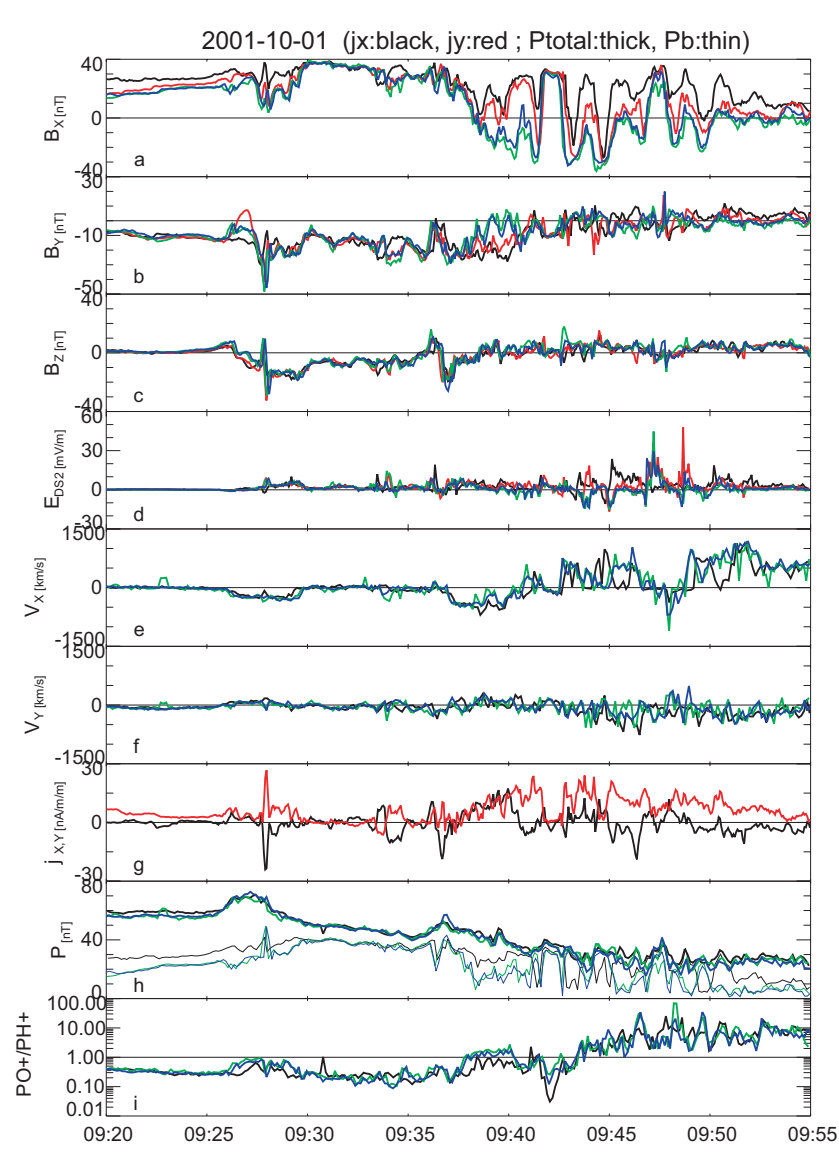

Fig. 9. Same format as Fig. 8 except for the bottom two panels, which are: (h) total and magnetic pressure, and (i) ratio between oxygen and hydrogen pressure. In the pressure plot the total and the magnetic pressures are plotted with thick and thin lines, respectively.

\subsubsection{October event}

Between 06:00 and 16:00 UT on 1 October, a series of semiperiodic substorms took place. The interval has a characteristic of a "saw-tooth" event during a large storm with a minimum SYM-H of 150 nT at 08:30 UT. The interplanetary magnetic field (IMF) was directed southward during the whole interval and ranged between $B_{z}=-15$ and $-2 \mathrm{nT}$. In this study we examine Cluster observations during the second substorm interval, when a LANL geosynchronous satellite (1991-080) detected multiple dispersionless electron and ion injections starting at 09:26 UT and a large substorm with $\mathrm{AE}>1000 \mathrm{nT}$ took place. As shown in Figs. $7 \mathrm{a}-\mathrm{c}$, Cluster was located at $X_{\mathrm{GSM}}=-16.4 R_{E}$, near $Z_{\mathrm{GSM}}=0$ in the premidnight magnetotail. The Cluster tetrahedron configuration at 09:20 UT is shown in Figs. 7g-i.

Figure 9 shows spin averaged field and particle data in same format as Fig. 8 except for the two bottom panels. The sum of particle and magnetic pressure is shown as a thick line and magnetic pressure is shown as thin line in Fig. 9h. 
Here the particle pressure was calculated using both proton and oxygen. We converted the pressure value into an equivalent magnetic field value (in nT), so that the likely lobe field strength can be inferred from the total pressure. The ratio between oxygen and hydrogen pressure is shown in Fig. 9i.

As can be seen from $B_{x}$ (Fig. 9a) and from the relative Cluster positions (Figs. 7e and f), the ordering of decreasing $B_{x}$ values, i.e., Cluster 1 , then 2 , then 4 , and finally 3 , is consistent with the relative order of the Cluster positions from north to south most of the time, suggesting that the tail current sheet orientation is approximately perpendicular to $Z_{\mathrm{GSM}}$ and that $B_{x}$ gives a good indicator of the location relative to the equator, on long time average. Yet, there are intervals with short-time perturbations or rapid current sheet crossings lasting less than a minute where the current sheet were significantly tilted from nominal orientation as will be discussed in more detail in Sect. 3.3. Cluster was initially located close to the northern lobe. Because of the high solar wind pressure and larger flux in the tail during a storm, the lobe field value during the initial interval is expected to be larger than $40 \mathrm{nT}$, as can be seen in the $B_{x}$ component when Cluster enters the lobe between 09:30 and 09:33 UT. After 09:37 UT, Cluster experienced several neutral sheet crossings until 09:53 UT, when all the spacecraft stayed in the plasma sheet. The first signatures of substorm disturbance at Cluster are the magnetic field fluctuations accompanied by tailward proton flow and encounter of the plasma sheet starting at 09:26 UT, which corresponds to the time of geosynchronous injection. After the plasma sheet encounter the total pressure started to decrease with some fluctuations and became $30 \mathrm{nT}$ by $09: 50$ UT and stayed nearly the same value afterwards. This negative trend in the pressure is a typical manifestation of unloading in the mid tail region during substorm expansion phase. As is often the case for a storm, the oxygen contribution is significant, as shown in Fig. 9i. Particularly after 09:44 UT the pressure is dominated by oxygen. This corresponds to the time interval of the thin current sheet as will be described later.

There were mainly two periods of enhanced tailward/earthward proton flows during the interval (Fig. 9e). The first one is the tailward flow between 09:26 and 09:30 UT near the geosynchronous injection time. Associated with this first tailward flow period (at about 09:27 UT), a sharp enhancement in $B_{y}$ and a positive, then negative disturbance in $B_{z}$ of about $30 \mathrm{~s}$ is observed, which is the typical signature of a tailward moving flux rope. The disturbance is accompanied by a large spike in the current density. This current is directly parallel to the ambient field flowing out from the ionosphere. Such $<30 \mathrm{~s}$ structures with $B_{z}$ reversals and $B_{y}$ perturbations are identified also during the next flow enhancement intervals.

The second flow interval is from 09:37 UT, and continues until 10:04 UT (not shown), with several flow reversals from tailward to Earthward and vice versa on a timescale of $>10$ min containing also rapid fluctuations. The $B_{z}$ pro- file in Fig. 9c also shows corresponding sign reversals on longer and shorter time scales: i.e., negative values on average, during predominantly tailward flow period and positive value mainly during Earthward flow periods, overlapped with faster fluctuations. The overall relationship between $B_{z}$ and flow, on greater than 10-min scale, is in a sense of producing dawn-to-dusk $\boldsymbol{V} \times \boldsymbol{B}$ electric field. Consistently, the dawnto-dusk electric field from EFW (Fig. 9d) became enhanced during the flow intervals exceeding several $\mathrm{mV} / \mathrm{m}$ (up to $10 \mathrm{mV} / \mathrm{m}$ ). Between 09:43 and 09:58 UT, even stronger electric fields were observed associated with neutral sheet crossings. Detailed field and plasma signatures between 09:46 and 09:51 UT, when strong electric fields, flow reversals and neutral sheet crossings were observed, will be discussed in Sect. 3.2.1.

Starting around 09:37 UT, when Cluster encountered the plasma sheet and observed tailward flow, persistent oscillations also started in the $B_{x}$ profile (Fig. 9a) with a time scale of about $2 \mathrm{~min}$. Based on minimum variance analysis of each crossing and timing analysis of the four spacecraft, these oscillations are due to a wavy current sheet. Assuming that the propagation vector identified from the current sheet crossings represents the motion of the current sheet, it is expected that the motion of the wavy current sheet is mainly duskward with a speed of $100-300 \mathrm{~km} / \mathrm{s}$. From this speed and the 2 min recurrence it is estimated that these wavy structure has a cross-tail spatial scale of $2-6 R_{E}$. It should be also noted that the inter-spacecraft difference in $B_{x}$ stands out during this period (Fig. 9a). The profile shows that the half thickness of the current sheet is expected to be smaller than the Cluster tetrahedron. The duskward current density obtained from the Cluster increases up to $20 \mathrm{nA} / \mathrm{m}^{2}$.

Another important observation for this event is the ion composition. During the thin current sheet interval, 09:4509:55 UT, pressure as well as density was dominated by $\mathrm{O}^{+}$ (Kistler et al., 2005), which was interpreted as being due to storm-time ion outflow. In this $\mathrm{O}^{+}$dominated thin current sheet, the $\mathrm{O}^{+}$ions were observed to execute Speiser-type serpentine orbits across the tail and were found to carry about $5-10 \%$ of the cross-tail current (Kistler et al., 2005). Detailed analysis of the distribution function showed separate $\mathrm{O}^{+}$layers above and below the thin current sheet (Wilber et al., 2004).

\subsection{Crossing of a quiet CS; 7 September 2001, 21:00 UT}

Figure 10 (panels a, b, and c), shows that $B_{x}$ changes from about $-20 \mathrm{nT}$ to $+20 \mathrm{nT}$, while $B_{y}$ and $B_{z}$ (plotted here with a different scale) and their fluctuations, are small (few nT). The current densities $J_{y}$, estimated by various methods are shown in panel (d). Since the current sheet is essentially perpendicular to $Z_{\mathrm{GSM}}$, we have fitted the $B_{x}$ component of the magnetic field measured by S/C 1 and 3 with a "Harris sheet model" (Harris, 1962) defined by $B_{x}=B_{L} \tanh \left(\left(z-z_{0}\right) / H\right.$ ) where $z_{0}$ and $H$ represent the center and the half-thickness 
of the current sheet, respectively. $B_{L}$ can be obtained either from direct measurement in the lobe region (if the $\mathrm{S} / \mathrm{C}$ happens to be located in the lobes) or by assuming the equilibrium of the vertical pressure within the plasma sheet (Baumjohann et al., 1990; Kivelson et al., 2005; Thompson et al., 1993). Here, for both periods, $B_{L} \approx 25 \mathrm{nT}$. Once these parameters are determined, we compute and plot the Harris current density at the equator (thick pink line), and at the location of S/C 3 (thick green line). We also plot the current density estimated from curl $B$ (thin pink line) and the contribution of the ions computed from CIS measurement on S/C 3 (thin green line).

Assuming that the current density profile is stationary during the crossing of the CS we find that $J_{y}$ is maximum near the center of the CS and that $J_{y_{\max }} \approx 10 \mathrm{nA} / \mathrm{m}^{2}$. The ion current is also quite close to the other estimates, which suggests that during the quiet crossing most of the current is carried by ions, in the s/c frame. The contribution of electrons to the current (not shown here) is indeed small. $J_{x}$ (not shown here) is much smaller than $J_{y}$ (panel d).

The fit with a Harris sheet has also been used to estimate the half-thickness of the CS $(H)$. In order to get $H$ we choose a couple of s/c with similar values of $Y$ and (if possible $X$ ), but different values of $Z$. This choice aims at minimizing the possible effects of radial and azimuthal modulations upon the determination of $H$. Figure $10 \mathrm{e}$ shows that, around 21:00 UT, $H \approx 2000 \mathrm{~km}$. During this period all the S/C are located inside the CS, hence the fit is good. The increases in $H$ found around 20:50 and 21:20 UT are probably not reliable, because the S/C get too close to the CS boundary. Figure $10 \mathrm{f}$ shows the position of the CS center $\left(Z_{0}\right)$, and the estimated location of its lower $\left(Z_{0}-H\right)$ and upper $\left(Z_{0}+H\right)$ boundaries, deduced from the same fitting procedure. It tells us that the CS moves southward at about $5.5 \mathrm{~km} / \mathrm{s}$, in the $\mathrm{S} / \mathrm{C}$ frame. Cluster spacecraft move slowly southward $(\sim 2 \mathrm{~km} / \mathrm{s})$; thus the CS moves at about $7.5 \mathrm{~km} / \mathrm{s}$. The motion of the CS can also be inferred from the time $(\sim 10 \mathrm{~min})$ it takes to cross the $\mathrm{CS}(2 \times 2000 \mathrm{~km})$. With these numbers we find that the CS center moves southward at about $7 \mathrm{~km} / \mathrm{s}$; consistent with previous estimate. Noticing that S/C 1, S/C 2, and S/C 4 are approximately at the same $Z_{\mathrm{GSM}}$, the delay between the crossing of the center of the CS can be used to characterize the flapping of the CS.

In summary a relatively thin CS (half thickness of about $2000 \mathrm{~km}$ ) can be stable over long time periods. For $N \sim \mathrm{cm}^{-3}, V_{t h i} \sim 1000 \mathrm{~km} / \mathrm{s}$, and $F_{H+} \approx 0.15 \mathrm{~Hz}$ (lobe field), we get the $\mathrm{H}^{+}$ion Larmor radius and ion inertial length $\rho_{i} \sim L \sim 1000 \mathrm{~km}$, i.e., half the CS half-thickness. As already mentioned, while describing Fig. 8, magnetic fluctuations are weak and no fast flow is observed during the crossing of this quiet but relatively thin CS. The slow flapping oscillation does not affect the stability of the CS.

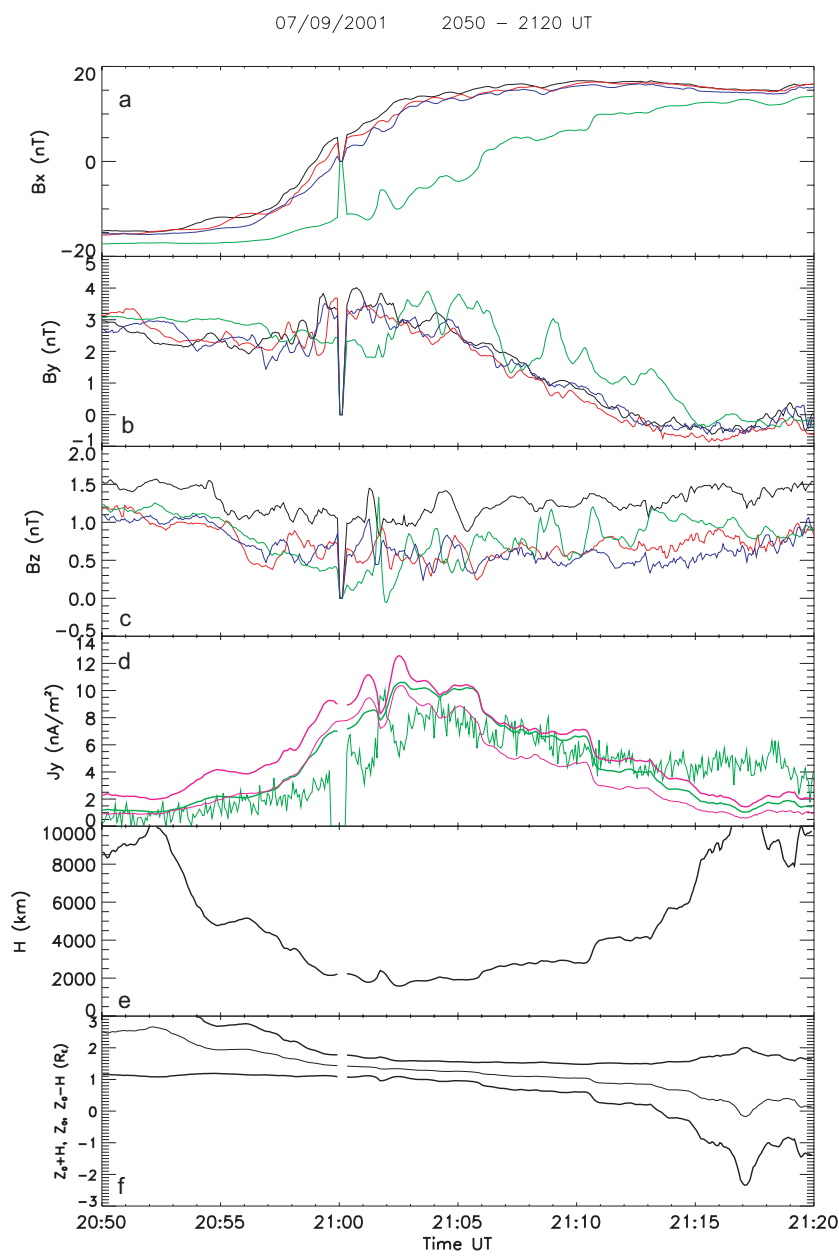

Fig. 10. Quiet CS crossing. Notice that $B_{y}$ and $B_{z}$ are not at the same scale as $B_{x}$; most of the magnetic field variations are on $B_{x}$, as expected for a 1-D CS. Panel (d) shows (i) Harris current density at the equator (thick pink line), (ii) at the location of S/C 3 (thick green line), (iii). $J_{y}$ from curlB (thin pink line) and (iv) contribution of ions to $J_{y}$, computed from CIS measurement on S/C 3 (thin green line); panels (e) and (f) show the half- thickness $(H)$ of the CS and the location of its center $\left(Z_{0}\right)$. Time resolution is $4 \mathrm{~s}$.

3.3 Crossings of an active CS, 7 September 2001, 21:3021:55 UT

\subsubsection{Magnetic fields and current densities}

The four top panels of Fig. 11 show again the $B_{x}, B_{y}, B_{z}$, the modulus of $B$, and the $-\mathrm{SR} 2$ component of the electric field (essentially $E_{y}$ ), with an enlarged scale. Magnetic and electric field data are now displayed with full resolution (22.4 points/s for $\mathbf{B}$ and 25 points/s for $E_{y}$ ). The averaged motion of the CS, as inferred from the averaged variations of $B_{x}$, is now upward. Panel (i) shows $J_{y}$, estimated from curl $B$. Until 21:33 UT curl B cannot be correctly estimated because all the S/C are still located outside the CS, or close to its boundary. After 21:33 UT the current density $J_{y}$ estimated from 


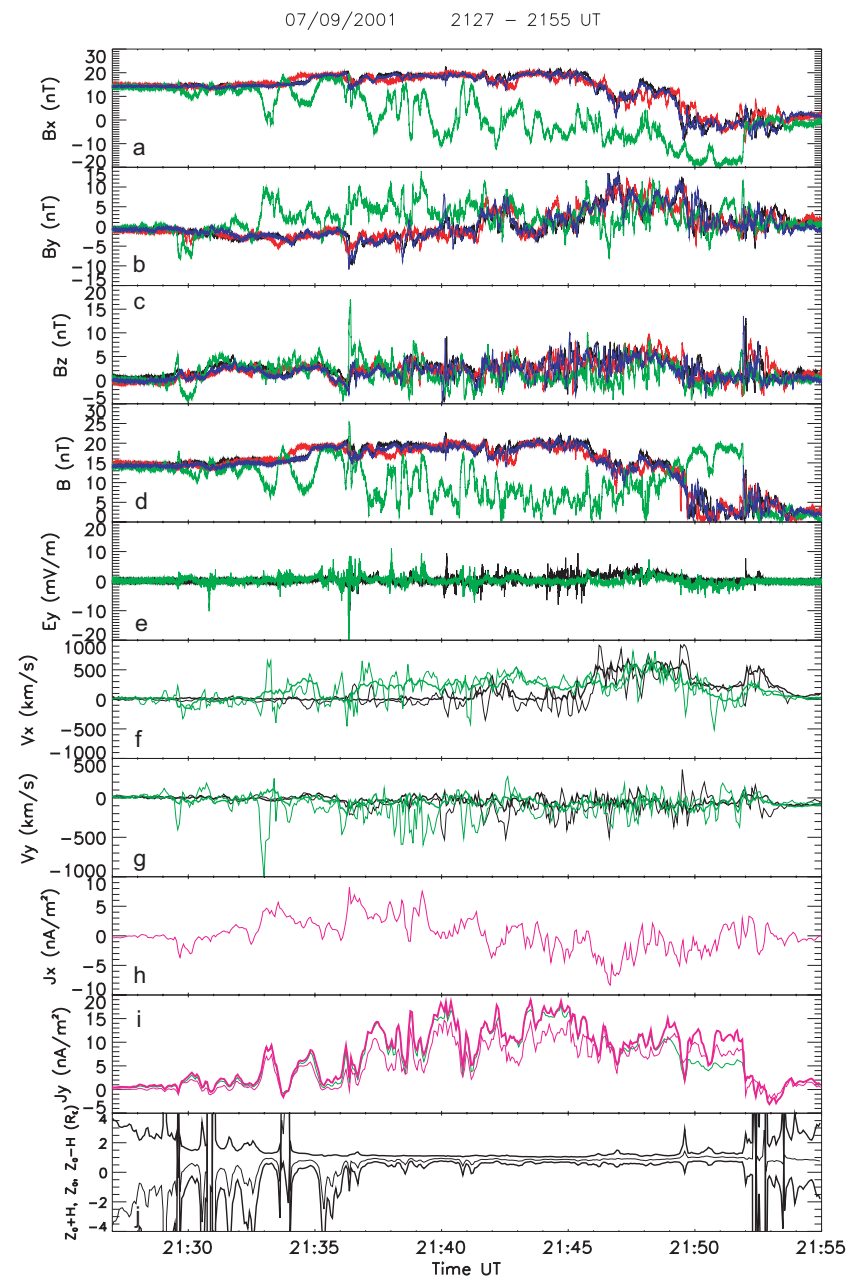

Fig. 11. Crossing of an active CS (September 7, 2001). From top to bottom, high time resolution data from FGM $\left(B_{x}, B_{y}, B_{z}, B\right)$ and EFW $\left(E_{y}\right)$. Unless otherwise noted, black is for S/C 1 , red for $\mathrm{S} / \mathrm{C} 2$, green for S/C 3 and blue for S/C $4 . V_{x_{i}}$ from CIS (thick line) and $V_{x}$ from PEACE (thin line) on S/C 1 and S/C 3, same for $V_{y}$,. $J_{x}$ and $J_{y}$ (thin pink line), computed from curlB, equatorial current density (thick pink line) and at the location of S/C 3 (thick green line) from Harris model (thin pink line), with $B_{L}=25 \mathrm{nT}$ (see text for more details).

the Harris fit and the current density computed from curl B agree quite well. Between 21:33 and 21:45 UT S/C 3, which is the only s/c located inside the CS, detects large amplitude, about $1 \mathrm{~min}$ quasiperiod fluctuations. The large amplitude fluctuations observed on $B_{x}(3)$ can be due (i) to a modulation of the total current $I_{y}$ below the S/C, (ii) to a flapping of the CS (with a large amplitude $\sim \mathrm{D}$ ), or (iii) to a modulation in the CS thickness $(H) . B_{x}$ at S/C 1, 2, 4 (outside the CS) being almost constant, interpretation (i) is ruled out. Thus the CS thickness is modulated (symmetric mode), or the CS flaps up and down (anti-symmetric mode), or a mixture of both. Whatever the mode, the fact that $B_{x}(3)$ can be negative, while $B_{x}(1,2,4)$ remain approximately constant and pos- itive, indicates that $H$ is comparable to, or even smaller than $D$. The fluctuations of $Z_{0}+H$ are much larger than that of $Z_{0}-H$ and $Z_{0}$ (see the last panel of Fig. 11), which suggests that the oscillations are asymmetric, or that $\mathrm{S} / \mathrm{C} 1,2,3$ are outside the CS, and therefore do not probe the fluctuations which are highly confined in the CS. In fact the LF modes can provide a proxy for the accuracy of the estimate of $Z_{0}$ and $H$. In the later case $Z_{0}$ and $H$ are not correctly estimated. For instance, around 21:35 UT, $H$ is probably largely overestimated and the largely negative value of $Z_{0}$ should not be trusted. Yet we can infer that between 21:33 and 21:45 UT, $H<D \approx 1500 \mathrm{~km}$, and that the total current inside the CS is essentially constant. Conversely after 21:45 UT, the three other S/C (S/C 1, 2, 4) penetrate in the CS; the estimates via the Harris fit are now accurate. A symmetric sausage like behavior is observed, with a constant $Z_{0}$ and symmetric variations of $Z_{0}+H$ and $Z_{0}-H$. Between 21:45 and 21:52 UT, where $H \approx 1500-2000 \mathrm{~km}$, large earthward flow bursts are observed (up to $600 \mathrm{~km} / \mathrm{s}$ ). After 21:52 UT, $H$ rapidly increases; $H \gg D$. At the scale of the tetrahedron, the magnetic energy has been dissipated since $B$ is quasi-null on all spacecraft. There are no longer large flow velocities. Thus the large amplitude quasi-periodic (60s) fluctuations are strongly confined in the CS and they develop only when the CS is thin, or very thin (21:33-21:45 UT). The thickening of the CS, and therefore the local dissipation of the magnetic energy, starts around 21:45 UT. After 21:52 UT, the CS gets very thick $\left(\sim 2 R_{E}\right)$; the magnetic energy has been dissipated and the transport of particles stops by 21:54 UT.

When the CS is thin or very thin, the fluctuations of $B_{y}$ and $B_{z}$ are quite large, in particular (but not only) on S/C 3. These fluctuations are interpreted as signatures of field aligned currents (see Sect. 4). Panels (h) and (i) show $J_{x}$ and $J_{y}$, estimated from curl $B$. Firstly, we observe a signature of negative parallel current $\left(J_{x}<0\right)$ between 21:29-21:30 UT associated with $V_{x}<0$ (tailward) and $V_{y}<0$ (dawnward), for electrons as well as for ions (see also Le Contel et al., 2002, and references therein), which suggests that the active region is localized earthward or westward of the S/C (see discussion in Sect. 4). In this current density structure the current is essentially antiparallel to $B$, and the spatial scale is comparable or smaller than $D$, as can be seen from the $B_{y}$ and $B_{z}$ profiles on S/C 3. Hence, the current density from the curlometer $\left(J_{x} \approx-5 \mathrm{nA} / \mathrm{m}^{2}\right)$ is probably underestimated. More generally, both $J_{y}$ and $J_{x}$ are likely to be underestimated, at least during the first period (21:33-21:45 UT). The fluctuations of $J_{x}$ (panel g) are as large as the fluctuations of $J_{y}$ (as expected from $\nabla \cdot J=0)$. Thus, unlike the previous crossing (at about 21:00 UT) the structure of the active CS is now 3D. The signatures of the FAC are seen on $B_{y}$, as expected, but also on $B_{z}$, which indicates that they have a small scale in the $\mathrm{Y}$ direction; they correspond to filamentary structures as will be shown in the next two figures. Notice that shorter period fluctuations $(T \sim 1-10 \mathrm{~s})$ are superimposed on the $\sim 60$ s fluctuations described above. Their amplitudes are quite large 

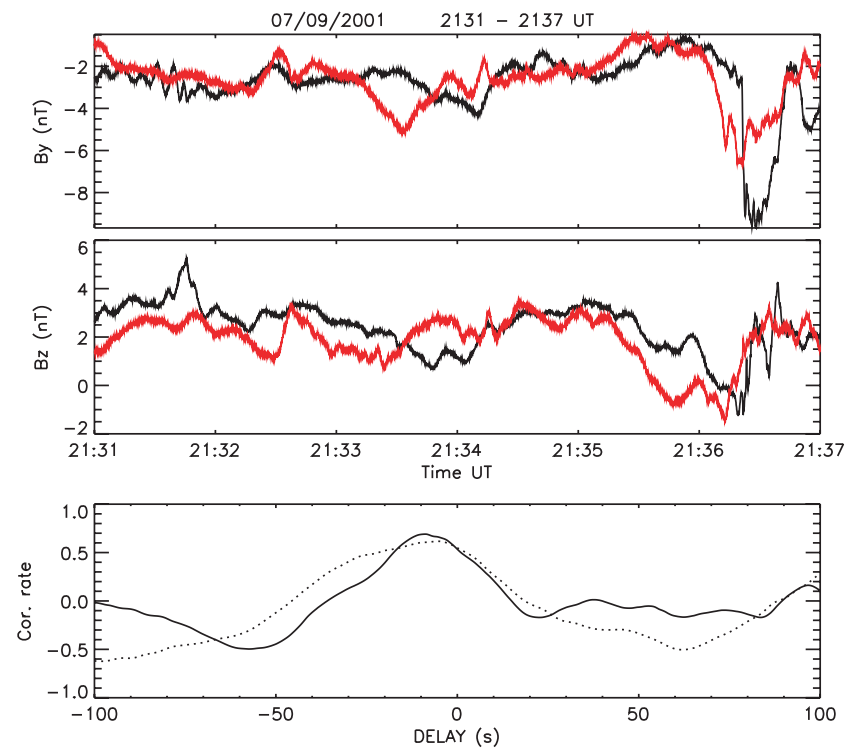

Fig. 12. $B_{y}$ and $B_{z}$ on $\mathrm{S} / \mathrm{C} 1$ and 2 at high-time resolution on 7 September between 21:31 and 21:37 UT. The full line is for the correlation function between $B_{y}(1)$ and $B_{y}(2)$, the dotted line gives the correlation function between $B_{z}(1)$ and $B_{z}(2)$. Time resolution of the correlation is $0.4 \mathrm{~s}$.

(about a few nT, $\sim 1-20 \mathrm{mV} / \mathrm{m}$ ), but still smaller than long period oscillations, at least for the magnetic components. We do not further discuss about these "high frequency" oscillations here.

In order to determine the direction of propagation of the CS fluctuations we correlate high time resolution data at $\mathrm{S} / \mathrm{C} 1$ and 2. The corresponding wave forms and correlation coefficients are displayed in Fig. 12. S/C 1 and 2 are essentially separated along $Y_{\mathrm{GSM}}$, by $\sim 2000 \mathrm{~km}$, while they are located at about the same $X_{\mathrm{GSM}}$ and $Z_{\mathrm{GSM}}$. Thus the delay obtained from the correlation lag $(\sim 10 \mathrm{~s})$ corresponds to an eastward motion at $\sim 200 \mathrm{~km} / \mathrm{s}$, in the same direction as electrons that carry the current in the S/C frame. In order to identify the 3-D characteristics of the fluctuations, we compare the four spacecraft magnetic field wave forms displayed in Fig. 13a (top panels). During this short time interval (40 s), $B_{y}(3)$ undergoes a positive excursion $(+10 \mathrm{nT}$ at 21:36:10 UT), while $B_{y}(1), B_{y}(2)$, and $B_{y}(4)$ get negative. The extrema of $B_{y}$, at the locations of the various $\mathrm{S} / \mathrm{C}$, are not simultaneous, as depicted by the vertical dashed lines. The delays between the extrema of $B_{y}$, at the various $\mathrm{S} / \mathrm{C}$, can be due to a radial or to an azimuthal motion. For S/C 1, 2 and 4 the dashed lines roughly correspond to zeros of $B_{z}$, but the maximum of $B_{y}(3)$, at 21:36:20 UT, does not coincide with a zero of $B_{z}(3)$. Instead it coincides with the maximum of $B_{x}(3)$. These observations are discussed in Sect. 4.2.1 where they are used to infer the shape and the motion of the corresponding structure.

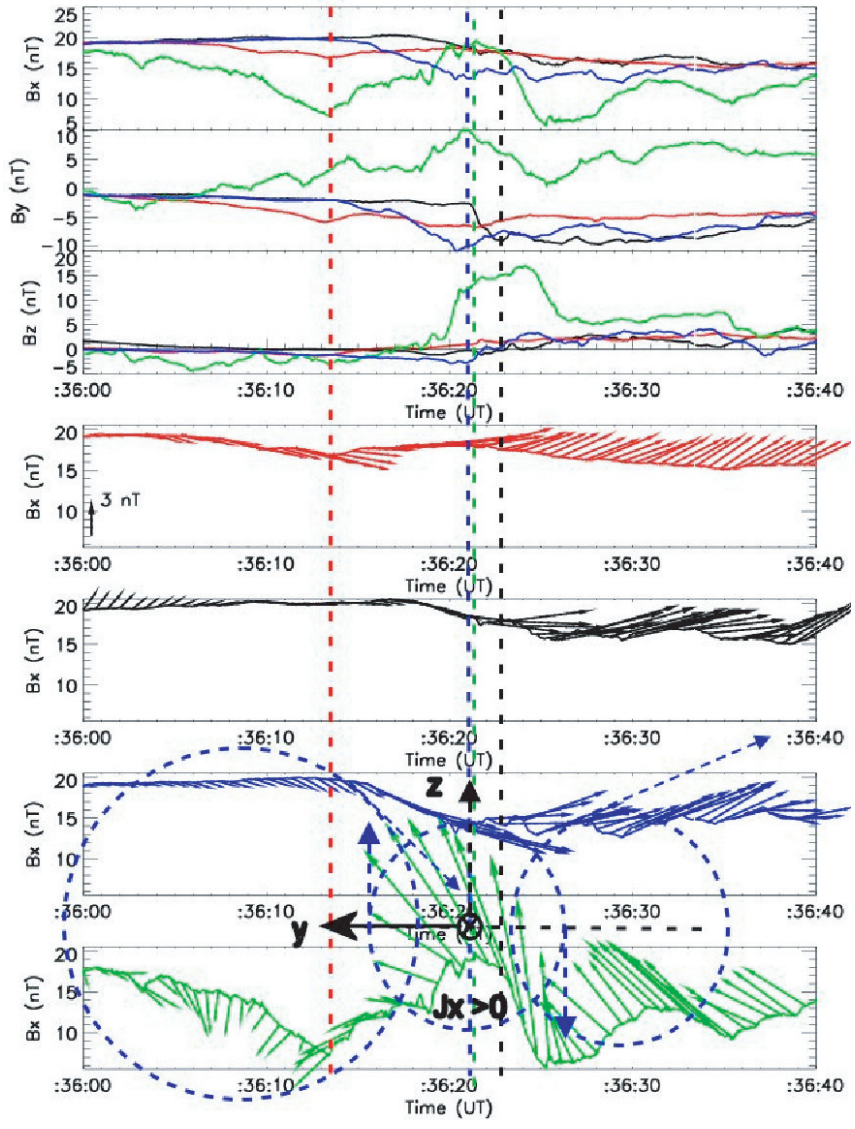

Fig. 13. (a) Magnetic field data between 21:36:00 and 21:36:40 UT, 7 September 2001. (b) Hodograms of the magnetic field projected onto the $(X Y)$ plane during the time interval shown in panel (a). Spacecraft are ordered by their locations along $Z$. $B_{X}$ is a proxy for the location of each S/C with respect to CS centre. Vertical dashed lines indicate the estimated closest approach, for each S/C. Dashed circles are drawn only for S/C4 (for clarity); they are tangent to magnetic field direction (in $B_{y}, B_{z}$ plane) taken at 3 selected times. To single out the magnetic field vectors corresponding with the selected times, we have extended their lengths via dashed arrows. Then the magnetic field direction is used for a remote sensing of the motion of the center of current tube. As discussed in the text the current tube is found to move eastward.

Figure 13b (4 lowest panels) shows hodograms of $B_{y}, B_{z}$ for the four S/C. $B_{x}$ is used as a proxy for the position of each $\mathrm{S} / \mathrm{C}$ with respect to CS center. Then the hodograms represent the projections of $B$ on the $\left(B_{y}, B_{z}\right)$ plane, as a function of time, at the location of the $\mathrm{S} / \mathrm{C}$, inferred from $B_{x}$. The hodograms are ordered by the position, along $Z_{\mathrm{GSM}}$, of the corresponding S/C, hence data from S/C 3 are displayed in the lowest panel. The amplitude of the modulations depends on how deep each $\mathrm{S} / \mathrm{C}$ is plunged into the CS. The largest fluctuations are observed at S/C 3, presumably because S/C 3 is closer to the center of the structure, but below, while the others are above or beyond. The hodograms give 


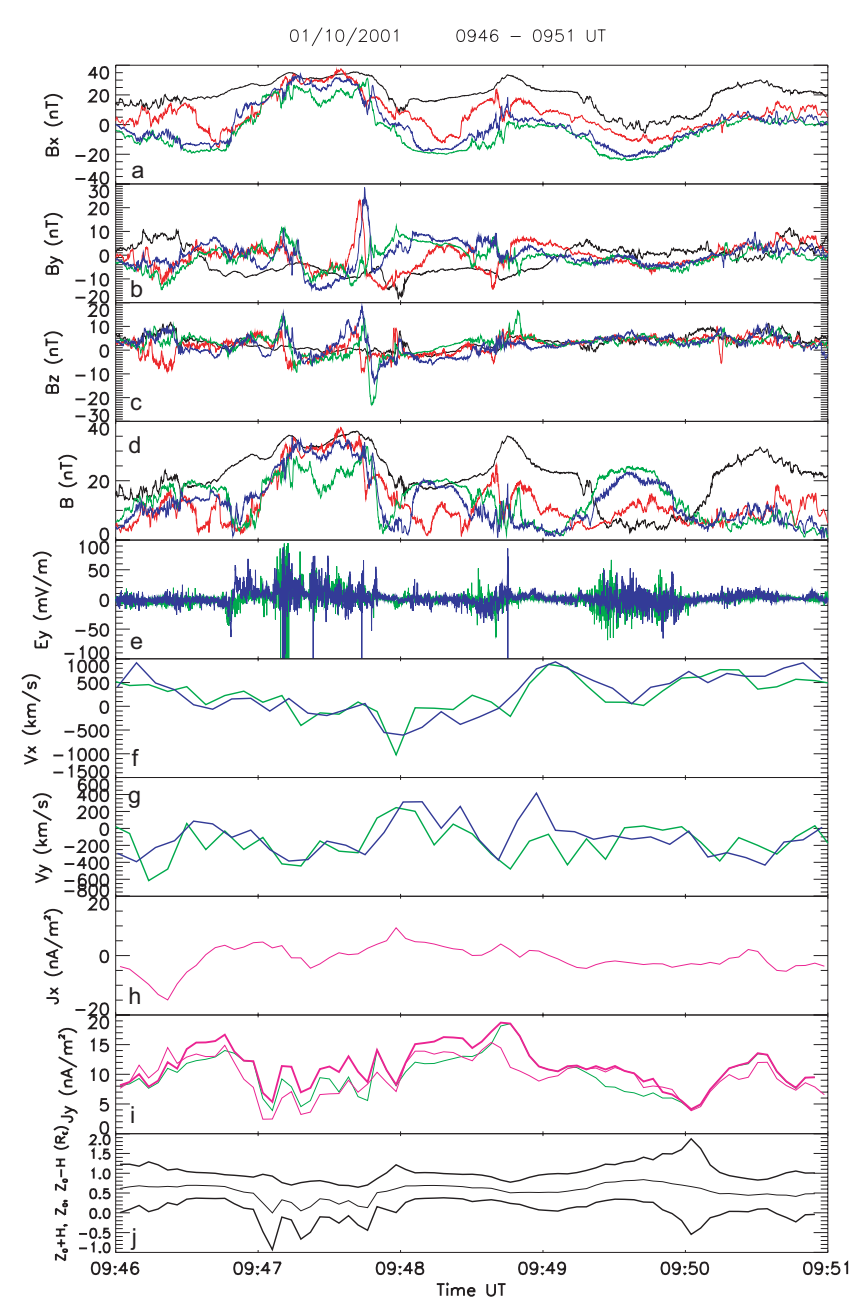

Fig. 14. (Flow reversal. Same format as Fig. 11. For this event $B_{L}=40 \mathrm{nT}$. The parameters of the Harris sheet are determined from $B_{x}$ measured by S/C 1 and 3, at different $Z$ and similar $X$ and $Y$. Panel (i) shows the two independent time profiles of $J$, estimated from Harris fit (thick pink,) and from curlB (thin pink). The green line is for $J$ at $\mathrm{S} / \mathrm{C} 3$, from Harris fit. CS parameters $\left(H\right.$ and $\left.Z_{0}\right)$ are determined from S/C 3 and 4. Data displayed in panels $(\mathbf{f}, \mathbf{g}, \mathbf{i}, \mathbf{j})$ are deduced from CIS/CODIF. Here time resolution of CIS data is $8 \mathrm{~s}$.

evidence for rotations of the magnetic field, with comparable amplitudes along $Y$ and $Z$, at least on S/C 3. In order to visualize the relation between $\delta B$ and a possible motion (radial/azimuthal) of the structure, we have selected three particular instances and used the corresponding magnetic field vectors (dashed arrows in 13b) to track the position of the center of the structure and to follow its motion. The results are discussed in Sect. 4.2.1.

\subsubsection{Ion and electron velocities}

Figure 11 shows the ion and electron velocities, computed from CIS and PEACE, for S/C 1 and S/C 3. The short lasting parallel current structure, around 21:29-21:30 UT, is associated with tailward ion and electron velocities, which can correspond to an active region developing earthward of the S/C. This is followed by bursty earthward ion and electron flow velocities $\left(V_{x i, e}\right)$ starting to develop first at S/C 3 (at about 21:33 UT), together with the fluctuations, as the spacecraft penetrates deep in the CS. The velocities are now earthward, suggesting that the active region is now tailward of Cluster. Large velocities are observed later at the other S/C, once they have penetrated in the CS (after 21:46 UT). Finally, between 21:50 and 21:52 UT, $B_{x} \approx-20 \mathrm{nT}$ at S/C 3; while $B_{x}$ close to zero at S/C 1, 2, 4, thus S/C 1, 2, 4 are now close to CS center while $\mathrm{S} / \mathrm{C} 3$ is near its southern boundary. Then $V_{x i}$ has moderate values at S/C 1, 4 and is small at S/C 3. Thus, in this thin and active CS, the ion velocity maximizes near the CS center and vanishes at its boundary. The average values of $V_{x e}$ follow $V_{x i}$, but short lasting bursts of $V_{x e}$ occur, with no ion counterparts.

In order to produce the westward current supporting the CS a substantial westward ion velocity $(\sim 100-200 \mathrm{~km} / \mathrm{s})$ is expected. As already shown by Fig. $8, V_{y_{i}}$ is small and negative; ions cannot carry the westward current. Figure $11 \mathrm{~g}$ shows that $V_{y_{e}}$ is negative and larger in absolute value than $V_{y_{i}}$; in average $V_{y_{i}}-V_{y_{e}} \approx 100-200 \mathrm{~km} / \mathrm{s}$, hence for $N \sim 1 / \mathrm{cm}^{3}$ we get $J_{y} \approx 15-30 \mathrm{nA} / \mathrm{m}^{2}$; somewhat above the estimate from curl $B$. Given that $\operatorname{curl} B$ underestimates $J_{y}$, a current density $J_{y} \approx 15-30 \mathrm{nA} / \mathrm{m}^{2}$ seems realistic. This value corresponds to $H \approx 500-1000 \mathrm{~km}$ which is comparable to, or smaller than, $\rho_{i}$, the ion Larmor radius $(\sim 1000 \mathrm{~km})$ in the lobe field. In Fig. 11 we see that the estimate of $J_{y}$ via curl $B$ is between the estimates (via a fit with an Harris sheet) of the current density at the equator and at S/C 3, thereby confirming the validity of these estimates; at least as long as $D \approx H$.

3.4 Active CS with flow reversals, 1 October 2001, 09:4609:51 UT

\subsubsection{Current sheet structures during rapid current sheet crossings}

Figure 14 shows the magnetic field, electric field, and ion data in the same format as Fig. 11, but during the second event, between 09:46 and 09:51 UT. Consecutive north-south excursions of the current sheet are observed during this interval with a time scale of about 1-2 min. By estimating the velocity of the current sheet motion using the temporal changes of $B_{x}$ and spatial gradient of $B_{x}$, profiles of the current density were reconstructed by (Runov et al., 2003, 2005) for these current sheet crossings, around 09:47:00, 09:48:00, 09:48:30, 09:49:30, and 09:50:00 UT and their spatial scale were estimated. Note that the flow reversal from Earthward to tailward took place around the first crossing, while a tailward to Earthward reversal took place around the third crossing. For the 09:47:00 and 09:48:30 UT crossings 
current sheet thickness was found to be less than the tetrahedron scale (Runov et al., 2003; Wygant et al., 2005), while it is broad $(\sim 4000 \mathrm{~km})$ or bifurcated during the 09:48:00, 09:49:30, and 09:50:00 UT crossings (Runov et al., 2003). While the parameter of the Harris-type current sheet gives generally a good indicator of the scale of the current sheet, these internal structures deviating from a Harris-type current sheet profile and the relatively large separation of the Cluster $\mathrm{S} / \mathrm{C}$ compared to these thin current sheet period could explain why the current sheet was estimated to be continuously thick at the bottom of Fig. 11 during this particular event.

The $B_{y}$ profile involves changes with time scale of the order of the duration of the current sheet crossing (as discussed above) and more transient peaks. During the first two crossings, when the flow is tailward, the general trend of $B_{y}$ is anticorrelated with that of $B_{x}$. On the other hand, after the 09:48:30 UT crossing, $B_{y}$ and $B_{x}$ profile during the crossing is correlated. In addition to this trend, there are transient peaks on a 10-s time scale, such as the one clearly seen on $\mathrm{C} 2$ and $\mathrm{C} 4$, around 09:48 UT. The same is true for $B_{z}$; transient variations are superimposed on a (longer time scale) negative $B_{z}$ during tailward flow and a positive $B_{z}$ during Earthward flow. Transient $B_{z}$ peaks or reversals are observed around 09:47:10, 09:47:45, 09:48:50 UT. The strongest transient peaks, on $B_{y}$ and $B_{z}$, are found around 09:47:45 UT; they will be later discussed in more detail.

Reversals of the electric field are associated with the crossings around 09:47:00 UT and 09:48:30 UT, during the thin current sheet crossings. The strongest electric fields were detected at the Northern Hemisphere, after the 09:46:50 UT crossing. Large amplitude fluctuations are also observed at much higher frequencies. "High frequency" electric fluctuations (up to $100 \mathrm{mV} / \mathrm{m}$ ) are shown in Fig. 14. Magnetic fluctuations, $\delta B^{2} \approx 1-3 \mathrm{nT}^{2}$, are also observed (not shown here) by STAFF. Furthermore, electrostatic waves with amplitudes $\sim 400 \mathrm{mV} / \mathrm{m}$ and frequencies varying from ion cyclotron to lower hybrid, and electrostatic solitary waves with amplitudes of $25 \mathrm{mV} / \mathrm{m}$ and much higher frequencies were observed by the Electric Field and Wave (EFW) instrument during 09:47-09:51 UT (Cattell et al., 2005).

Energetic electrons (a few $\mathrm{keV}$ ) are observed when the spacecraft is inside the CS, as monitored by the modulus of $B_{x}$ (see Fig. 15). Outside the CS proper, in the CS Boundary Layer (CSBL), large fluxes are still observed, but at much lower energies; less than $1 \mathrm{keV}$. Notice also that the electron flux is very anisotropic; in the CSBL the electron flux (below $1 \mathrm{keV}$ ) is much larger in the parallel (bottom) and antiparallel (top) directions than in the perpendicular (middle). Hence CSBL electrons show bi-directional electron fluxes. Disregard the lowest energy channel which is contaminated by photoelectrons. Interesting to note that the most energetic electrons are detected during the transient $B_{z}$ events described before.

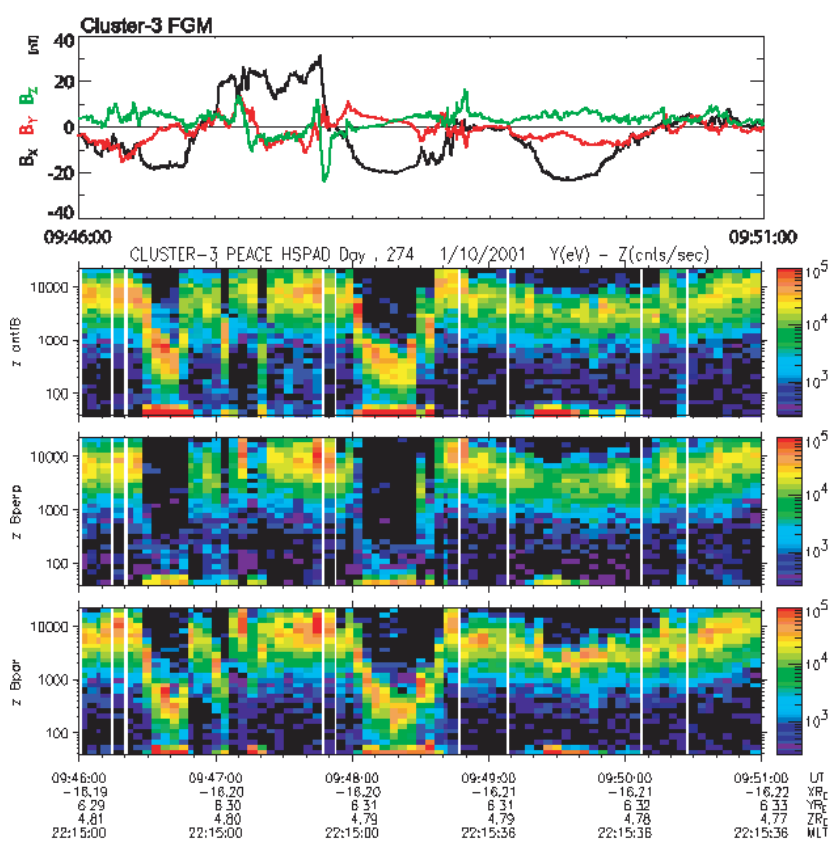

Fig. 15. $B_{x}, B_{y}, B_{z}$ profile (from FGM) and spectrogram showing the electron flux (from PEACE), color coded, versus energy and time, for S/C3 during the same time interval as Fig. 14. Time resolution is $4 \mathrm{~s}$.

\subsubsection{Transient $B_{z}$ reversals}

Short duration $B_{y}$ and $B_{z}$ enhancements with reversals on $B_{z}$ are observed between 09:46-09:51 UT. The most prominent structure occurs at 09:47:45 UT. Figure 16a is a blowup showing this structure at an enlarged scale. S/C 1, the northernmost $\mathrm{S} / \mathrm{C}$ hardly detects the signature of the structure. This lack of detection suggests that the structure has a small size along $Z$, and is located well below $\mathrm{S} / \mathrm{C} 1$. This is confirmed by the differences between the signatures at different $\mathrm{S} / \mathrm{C}$; the size of these structures should be smaller than the distance between the $\mathrm{S} / \mathrm{C}(\sim 1500-2000 \mathrm{~km})$, at least along $Z$. The signature of the structure involves a large positive excursion of $B_{y}$, at S/C 2 and S/C 4, with very similar temporal profiles. The delay between the $B_{y}$ signatures at $\mathrm{S} / \mathrm{C} 2$ and S/C 4 suggests a propagation of the structure, as discussed in Sect. 4.3.2. The maximum of $B_{y}(2)$, red dashed line, is associated with a reversal in $B_{z}(2)$. There is also a reversal in $B_{z}(4)$, but there is a small time shift between the zero of $B_{z}(4)$ and the maximum of $B_{x}(4)$. Unlike $B_{y}(2)$ and $B_{y}(4)$, $B_{y}(3)$ does not yield a large excursion. Yet $B_{z}(3)$ shows a clear reversal, from positive to negative, with an amplitude as large as for S/C 4 and larger than for S/C 2. A large positive excursion of $B_{x}(3)$ is observed at the same time as the reversal in $B_{z}(3)$. It is interesting to note that although position in $Z$ and plasma data suggests $\mathrm{C} 3$ should be closer to the equator, $B_{x}$ is larger at $\mathrm{C} 3$ than at $\mathrm{C} 2$ and $\mathrm{C} 4$ indicating that the current sheet appears to be filamented. Figure 16b 


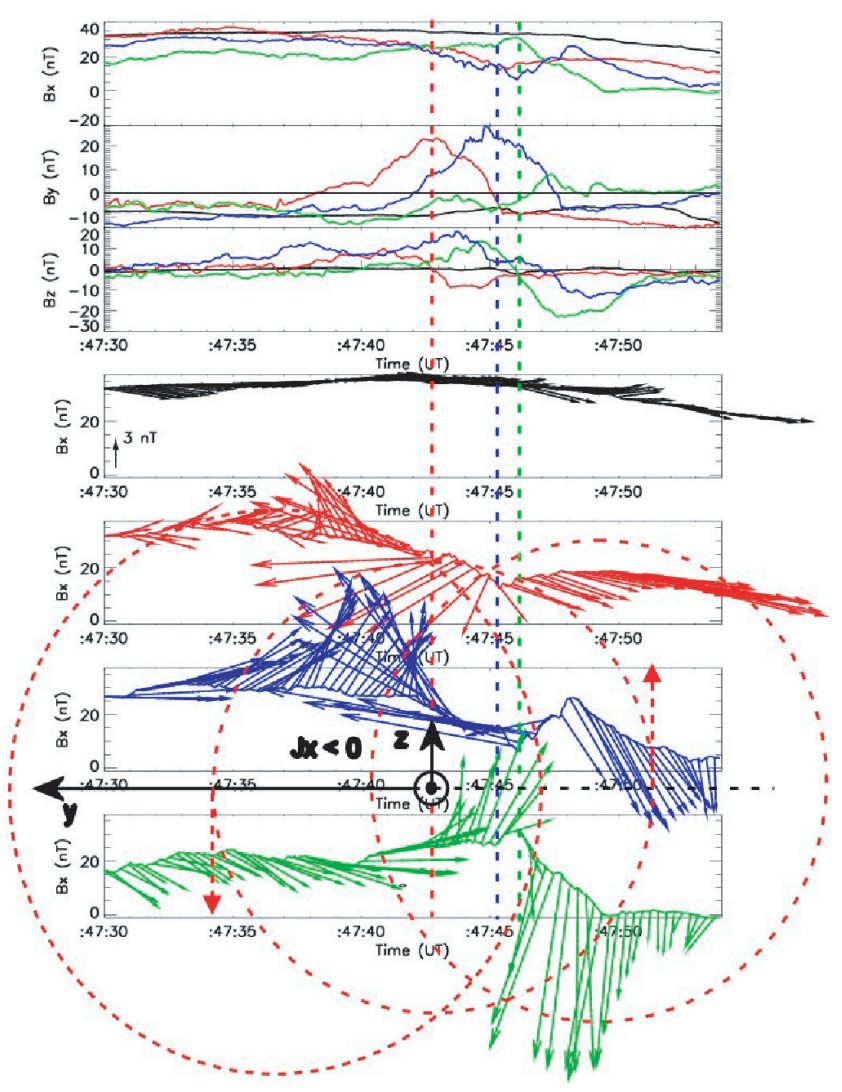

Fig. 16. 1 October 2001, event, same format as Fig. 13. Notice that $B_{y}(4)$ can be deduced from $B_{y}(2)$ via a time shift of $\sim 5 \mathrm{~s}$ (a). On (b) dashed red circles are tangent to the magnetic field measured by $\mathrm{C} 2$, at 3 successive times. Dashed lines are intended to single out the directions of the $B$ for these instances.

further illustrates the nature of the magnetic field structure at 09:47:45. The same presentation as for Fig. 13b is used. Spacecraft are again ordered by their positions along $Z_{\mathrm{GSM}}$. Using $B_{x}$ as a proxy for the distance with respect to the CS center, we find large rotations of the vector $B$, projected onto the (YZ) plane. As for Fig. 13b, the dashed circles have been built as tangent to the magnetic field vector at $\mathrm{C} 2$, taken at 3 arbitrary times indicated by dashed arrows. A detailed interpretation of Figs. 16a and $\mathrm{b}$ is given in Sect. 4.3.2.

\section{Discussion and interpretation}

After a short summary of the key observations, the two events, 7 September 2001 (Event 1) and 1 October 2001 (Event 2), are discussed and interpreted based on different models.

\subsection{Event 1: Summary of key observations}

(i) The first crossing (at about 21:00 UT) corresponds to a relatively thin $\mathrm{CS}, H \sim 2000 \mathrm{~km}\left(\sim 2 \rho_{i}\right)$, where $H$ is the half
CS thickness and $\rho_{i}$, the ion Larmor radius, and with a high $\beta \sim 100$. Yet, during this crossing, the CS is quiet; neither fast flows nor large amplitude fluctuations are observed. For this moderately thin CS, westward drifting ions carry most of the westward current.

(ii) During the subsequent period of crossings (21:3321:45 UT), $H \leq D$, the distance between the spacecraft $(D \approx 1500-2000 \mathrm{~km})$. Short lasting bursts of moderately fast earthward ion flows $(\sim 300 \mathrm{~km} / \mathrm{s})$ are observed only by S/C 3, near the CS center. Later, between 21:45 and 21:52 UT, while $H$ increases, fast earthward ion flows $(\sim 600 \mathrm{~km} / \mathrm{s})$ are observed by all $\mathrm{S} / \mathrm{C}$, together with large amplitude fluctuations ( $\sim \sim 1 \mathrm{~min})$. Magnetic fluctuations are highly confined in the CS and the flow velocity maximizes near the CS center This active CS persists and remains thin for about $20 \mathrm{~min}$, well after the start of the large amplitude fluctuations at Cluster (21:30 UT) and the development of the auroral bulge (at about 21:29 UT from Polar and IMAGE). Transient increases of $B_{z}$ and $H$ (transient dipolarization) occur, for instance around 21:34 UT, but a long lasting increase of $B_{z}$ and $H\left(H \approx 2 R_{E}\right)$, and hence a large decrease of $J_{y}$, only occurs around 21:52 UT.

(iii) During the active period $V_{y_{i}}$ is small and negative (about $-50 \mathrm{~km} / \mathrm{s}$, on average), hence ions cannot carry the westward current. Electrons have large negative velocities, $V_{y_{e}}$. This fast eastward drift of electrons can be related to an electric field $E_{z}$ pointing towards $\mathrm{CS}$ centre. Then electrons drifting eastward faster than ions can carry the current in this thin active CS (see also Asano et al., 2004). Bursts of fast eastward electron drift can also be associated with a modulation of the thermal anisotropy of electrons $\left(T_{\|}>T_{\perp}\right)$. Indeed the current being maximum near the equator, the curvature radius is very small near CS center, and electrons could also exhibit strong curvature drift. Ions are less sensitive to strong curvature effects because they are not adiabatic. Effectively, during the active period, the electron distributions (not shown here) are often very anisotropic $\left(T_{\|} \gg T_{\perp}\right)$, thereby providing an alternative/complimentary mean of carrying the $J_{y}$ current, as suggested by Mitchell et al. (1990).

(iv) On average, the radial velocity $V_{x e} \approx V_{x i}$; thus we can really speak of a flow. Yet the instantaneous value of the electron flow velocity generally does not match that of ions; it is much more fluctuating. This is indicative of small scale field aligned currents (FAC). The corresponding $J_{x}$ has the same order of magnitude as $J_{x}$ from curl $B$. The magnetic signatures of these small scale field aligned currents (FAC) are seen on $B_{y}$ (as expected), but also on $B_{z}$. 


\subsubsection{Event 1; interpretation of CS signatures with $\mathrm{CD}$ model}

Point (ii) is puzzling; we knew that a very thin CS can persist for quite a long time (see, for instance, Mitchell et al., 1990). Around 21:00 UT we do observe a relatively thin quiet CS that lasts a long time, but present observations show that an active CS (with fast flow and large amplitude fluctuations) can also last $20 \mathrm{~min}$; Event1 is an example of such a "metastable" thin CS. What is the nature of the corresponding fluctuations, and what causes the observed flow?

\section{Nature of the fluctuations}

S/C located inside the active CS detect large amplitude fluctuations together with fast flow bursts. Yet the magnetic field components $B_{x}$ measured by S/C located outside, or near the CS boundary, weakly vary, which indicates that (i) the total current inside the CS is conserved, and (ii) the fluctuations are highly confined in the CS. Therefore these fluctuations correspond to oscillations in the spatial distribution of the current density inside the CS, associated with symmetric or antisymmetric modes of the CS, as expected for ballooning modes and cross-field current instability. Both are expected to propagate azimuthally. As described in Sect. 3.2.1. The delay, obtained from the correlation lag $(\sim 10 \mathrm{~s})$ between SC 1 and SC 2, corresponds to an eastward motion at $\sim 200 \mathrm{~km} / \mathrm{s}$, in the same direction as electrons that carry the current in the S/C frame. Then if we interpret the observed fluctuations as ballooning modes, they have to move eastward. At first glance this is surprising, since drift ballooning are expected to move westward, with drifting ions. Yet the proper frame for studying ballooning modes (as other modes) is the quasi-neutrality frame (see discussion in Sect. 2.1) where the equilibrium electrostatic potential is null (if such a frame can be found). For instance in the case of a Harris equilibrium it is defined by $V_{y_{i}} / T_{i}+V_{y_{e}} / T_{e}=0$ (Harris, 1962). In a situation where the current is carried by electrons and $T_{i} \gg T_{e}$ (here $T_{i} / T_{e} \approx 5$ ), the quasi-neutrality frame is very different from the $\mathrm{S} / \mathrm{C}$ frame; it is moving at $V_{Q N} \sim V_{y_{e}}(<0)$. In that frame (the $\mathrm{QN}$ frame) the ions and the associated ballooning modes are moving westward, as expected for driving unstable drift ballooning modes satisfying approximately $\omega \approx k_{y} V_{y_{i}}$. Thus the observed magnetic fluctuations are likely to correspond to ballooning modes. What is the relation between these modes and the structure of the currents.

\section{3-D structure of the currents}

As discussed in the theory section, ballooning modes produce an azimuthal modulation of the $J_{y}$ current, coupled via $\nabla \cdot \mathbf{J}=0$, to localized field aligned currents $\left(J_{x}\right)$. The expected signature of ballooning modes is shown in Fig. 5; it consists in a modulation of $B_{x}$ (associated with an azimuthally traveling modulation of $J_{y}$ ), and a modulation of $B_{y}$ and $B_{z}$ (as- sociated with the passage of azimuthally traveling filamentary current structures, $J_{x}$ ). This is essentially what Fig. 11 shows: quasi-periodic fluctuations of $B_{x}$, together with fluctuations in $B_{y}$ and $B_{z}$. On the other hand, the NENL model does not require variations along $Y\left(k_{y}>0\right)$. If $J_{y}$ was more or less azimuthally invariant, as expected for a CS structured by a quasi-steady NL, the direction of the magnetic field vector, projected on the $\left(B_{y}, B_{z}\right)$ plane, would remain along a given direction, and the sign of $B_{z}$ would reverse only while passing by a radially moving NL.

Detailed information about the structure of filamentary currents has been obtained by zooming in some of them (see Fig. 13 and the corresponding discussion). Figure 13 (top panels) shows that $B_{y}(3)$ undergoes a positive excursion $\left(+10 \mathrm{nT}\right.$ at 21:36:20 UT), while $B_{y}(1), B_{y}(2)$, and $B_{y}(4)$ undergo negative excursions. S/C 3 being at a lower $Z$ than its companions, these opposite senses of variations indicate that a current density structure is located between S/C 3 and its companions. Given the respective locations of the S/C (see Fig. 7) the delays between the extrema of $B_{y}$ can be interpreted as evidence for an eastward motion of the current structure. Indeed, S/C 2 is at about the same $Z$ as S/C 1 and $\mathrm{S} / \mathrm{C} 4$, but at a larger $Y$. Hence S/C 2, which is to the west of the others, is the first one to detect the eastward moving structure. The dashed lines, built from the extrema of $B_{y}$, or more precisely from the mid-values of the corresponding positive or negative excursions (neglecting short lasting fluctuations), roughly correspond to the zeros of $B_{z}(1), B_{z}(2)$, and $B_{z}(4)$, as expected for an eastward moving cylindrical current density. The fact that the zero of $B_{z}(3)$ does not coincide with the maximum in $B_{y}(3)$ can be due to a residual $B_{z}$, superimposed, or to a local dipolarization affecting primarily the S/C which is closer to the center of the CS. Anyhow, the maximum of $B_{x}(3)$, at 21:36:20 UT, coincides with the maximum of $B_{y}(3)$, which is consistent with flux rope models (see, for instance, Khurana et al., 1995), where the magnetic field component along the axis of the tube, here $B_{x}(3)$, is maximum at the centre of the cylindrical structure. The observed magnetic wave forms are therefore consistent with being the signatures of a more or less cylindrical current density structure moving eastward. In this structure the current is essentially along the $\mathrm{X}$-direction, and the closest approach occurs at 21:36:22 UT, for S/C 3. Figure 7 shows that the distance between S/C 2 and S/C 1 (projected along $Y$ ) is about $1800 \mathrm{~km}$. Given that the corresponding delay is $9 \mathrm{~s}$, the structure moves at $\sim 200 \mathrm{~km} / \mathrm{s}$ eastward.

Figure 11 shows that the flow velocity is often very unsteady; short lasting bursts are detected only by the S/C that happen to be located inside the CS. Yet, unsteadiness does not only result from entrance/exit in/from the CS. Indeed when two or more S/C are located inside the CS, (as it is the case around 21:50 UT) they detect simultaneously flow bursts. Therefore the time variability of flow bursts cannot simply be due to fluctuations in the location of the S/C with respect to the location of the CS centre. According to the 
CD model enhanced flow bursts are due to sudden decreases of the local current density, associated, via $\nabla \cdot \mathbf{J}=0$, to fieldaligned current structures. Indeed $\partial J_{y} / \partial t$ is related to $E_{y}$, and hence to $V_{x}$, via $\mu_{0} \partial J_{y} / \partial t \approx \partial^{2} E_{y} / \partial z^{2}$, which is valid for a thin CS, and for $\nabla \cdot \mathbf{E} \approx 0$ (see theory section). Thus fast decreases in $J_{y}$ produce bursts in $E_{y}$ and hence fast flow bursts. A fast decrease of $J_{y}$ can either lead to an earthward or to a tailward flow burst, depending on the sign of $B_{z}$. Examples of ions flows bursts, associated with positive excursions of $B_{z}$ and increases in CS thickness $(H)$ are found near 21:36:20, 21:41:30, 21:45:40, and 21:52 UT (see Fig. 11), but the $B_{z}$ increase is short lasting.

\section{Quasi-steady transport of the plasma}

However a quasi-steady radial flow is often observed, superimposed on the faster transient flow bursts discussed above. This quasi-steady flow is likely to result from the cumulative effects of fluctuations generated beyond $\left(B_{z}>0, V_{x}>0\right)$, or inside of (for $\left.B_{z}<0, V_{x}<0\right)$ ) Cluster. To estimate the role of the fluctuations in the quasi steady earthward transport we can use a paper by Chen (1999), who gives a quasi-linear description of the transport by large amplitude fluctuations. He shows that in addition to the classical diffusion terms, there is a net radial transport associated with azimuthally moving fluctuations $\left(k_{y}\right)$. It is important to realize that the direction of propagation of the low-frequency fluctuations (in the plasma frame) determines the direction of the radial transport. Fluctuations displayed in Fig. 11 have very large amplitudes, $\left(\delta B_{\|} / B \sim 1\right)$, hence a relatively large transport is to be expected. Using formula 54, from Chen (1999), and the following parameters: $f_{i} \approx 0.2-0.4$ ( $f_{i}$ being the proportion of resonant ions), $V_{A} \approx V_{t h i} \approx 1000 \mathrm{~km} / \mathrm{s}, \lambda_{y} \approx 10000 \mathrm{~km}$, $B \approx 20 \mathrm{nT}$ and hence $\rho_{i} \approx 1000 \mathrm{~km}$, we find that the net flow velocity inward is $V_{x} \approx 200-400 \mathrm{~km} / \mathrm{s}$, consistent with the observed quasi-steady flow. Hence at least part of the earthward transport can be relatively steady and accounted for by a quasi-linear description, such as the one described above. This interpretation is consistent with the observation of large amplitude fluctuations which are regularly associated with fast flows.

Hence, during the active phase, ballooning modes which are observed to be confined in the CS, can possibly account for bursty flows associated with localized current disruptions, and/or quasi-steady flow, when the effect of these local accelerations is integrated over a broad region.

The CD scenario has been used to interpret CCE observations carried out at $7-9 R_{E}$, but there is nothing in the model that prevents its applicability to regions located at larger distances. Indeed, Image data show that Event 1, which is probably a pseudo- breakup, develops only at relatively large latitudes of $>70^{\circ}$ (H. Frey, personal communication), i.e., at relatively large distances from the Earth.
4.1.2 Event 1: interpretation of CS signatures with NL model

The observed thin current sheet interval is associated with fast, predominantly Earthward, flow with bursty enhancements in $B_{z}$. These Earthward flows suggest that the reconnection region was tailward of the spacecraft. While the entire enhancement of the current sheet has a time scale of 20 min, each flow enhancement has a time scale of minutes and is accompanied by bursty $B_{z}$ enhancements. Burst signatures of the plasma sheet flows or magnetic disturbances at the boundary of the plasma sheet have been interpreted as being due to temporal variations in reconnection or to spatial structures of the reconnection region, so that we are possibly observing consequences of remote reconnection sites. Transient $B_{z} / B_{y}$ features, for example, have been interpreted as due to flux rope/plasmoid due to multiple reconnection region (Slavin et al., 2003) or night side flux transport event caused by bursty reconnection (Sergeev et al., 2005), or due to field-aligned current as a consequences of the shear in the fast flows localized in the Y direction (Nakamura et al., 2005).

The flow seems to be centered near the center of the plasma sheet. Yet, magnetic signatures at the edge of the plasma sheet suggest that these flows could be accompanied by Earthward directed current filaments confined to the edge of the plasma sheet as shown in Fig. 13. In Fig. $11 J_{x}$ is mainly Earthward until around 21:42 UT and then turns to mainly tailward, when all the spacecraft eventually covers the center of the plasma sheet. This change in the direction of $J_{x}$ is consistent with the closure of the Hall-current in the reconnection region. Although the current sheet thickness of Event 1 is slightly larger than a typical ion scale, this closure current mainly along the magnetic field was predicted to be observable even away from the ion diffusion region based on theoretical studies (Treumann et al., 2006). In particular, the observations in Fig. 12 show that the contribution to the current corresponding to the outer edge of the plasma sheet is consistent with an inflow current toward the reconnection region. Yet, the bursty features as well as the 3-D profiles seen in Fig. 13 suggest that the $J_{x}$ pattern due to an X-line tailward of the spacecraft is obtained only in an average sense and that temporal/3-D processes are essential.

\subsection{Event 2: Summary of key observations}

Event 2 is an example of an active storm-time substorm current sheet. Cluster observed multiple current sheet crossings associated with flow reversals. The key observations can be summarized by sorting out the disturbances at different time scales.

(i) Substorm expansion phase ( $\sim 30$ min) During the first signature of the substorm injection at geosynchronous orbit, around 09:26 UT, Cluster was still near the CS 
boundary, but the total pressure starts to decrease gradually and continues to decrease during the subsequent thin current sheet intervals. Enhanced current densities were observed for about $20 \mathrm{~min}$. It is interesting to note that this time scale of the current density enhancement is comparable to Event 1.

(ii) Flow reversal ( $10 \mathrm{~min})$ Tailward flows associated with negative $B_{z}$ and Earthward flow associated with positive $B_{z}$ are detected. They overlap with shorter-time scale disturbances as discussed below. Mainly two such short lasting reversals were detected during this interval.

(iii) Current sheet flapping (a few minutes) The observed large amplitude fluctuations are moving azimuthally eastward and correspond to a flapping of the CS; hence the corresponding mode, for the current density, is antisymmetric. The active period (09:46-09:51 UT) described above (Fig. 12) corresponds to a thinning of the current sheet, superimposed on the flapping; current sheet profile estimated from a fit with an Harris current sheet, and from the flapping of the current sheet, show that $H$ decreases from $\sim 3500 \mathrm{~km}$ to $\sim 1500-2000 \mathrm{~km}$ or even thinner. As for Event 1, the large amplitude oscillations $(T \sim 100 \mathrm{~s})$, observed during Event 2, correspond to a spatial modulation of the tail current $J_{y}$. Systematic By changes are observed in association with the current sheet crossings and with (anti-)correlation of $B_{x}$ and $B_{y}$ during (Earthward) tailward flow interval.

(iv) Transient $B_{z} / B_{y}$ peaks/reversals (a few, up to $10 \mathrm{~s}$ ) Large amplitude fluctuations are observed on $B_{y}$ and $B_{z}$, in particular around 09:47, 09:48, and 09:49 UT; the corresponding 3-D structure can be deduced from the displays shown in Fig. 16. Accelerated electrons are observed in association with these transient 3-D structures.

(v) Higher frequency fluctuations (less than 1 s) Large amplitude fluctuations are also observed at much higher frequencies up to $10 \mathrm{~Hz}$ (the maximum frequency for wave form transmission in the nominal mode), and beyond such as solitary waves in the PSBL region.

\subsubsection{Event 2: Interpretation with NENL model}

During the time scale of a substorm, this event shows reversals from a negative $B_{z}$ associated with tailward flow in the plasma sheet to a positive $B_{z}$ and Earthward flow, as expected for an $\mathrm{X}$-line configuration associated with reconnection. The second tailward-to-Earthward flow reversal between 09:47 and 09:51 UT has been interpreted by Runov et al. (2003) as an evidence for a tailward moving X-line passing near the $\mathrm{S} / \mathrm{C}$, based on flow $/ B_{z}$ relationships, direction of the curvature obtained from the current sheet crossing. Multiple reversals of the flow are either due to the backand forth motion of this $\mathrm{X}$ line, or to different $\mathrm{X}$-line that may be activated. Another strong evidence for X-line configuration during this interval is that By showed systematic changes during current sheet crossings, differently for tailward and Earthward flow as discussed in Sect. 3.3.1. This quadrupolar out-of-plane magnetic field component can be interpreted as a manifestation of the Hall current system, resulting from ion-electron decoupling within the ion diffusion region with the characteristic scale of the ion inertial length. Simultaneous multi-point observations by Cluster show the spatial structure of the Hall region and also confirm that the current sheet thickness is in fact less than an ion (oxygen) scale (Runov et al., 2003, 2005). Furthermore, consistent electric field directed toward the center of the current sheet was identified via $E_{y}$, thanks to the fact that for this event the current sheet is sometimes largely tilted in the $Y Z$ plane (Wygant et al., 2005).

Overlapped with this large-scale trend, peaks or reversals in $B_{z}$ are detected, with a time scale of several to $10 \mathrm{~s}$ and associated fast flows. These events can be interpreted as consequences of reconnection, reflecting temporal changes in the reconnection rate and/or spatial structure of the reconnection region or due to interaction with fast flows and ambient fields. The 09:47:45 UT event is another example that such features are spatially limited both in $Y$ and $Z$ direction but also limited in time to about $10 \mathrm{~s}$, and can be multiple features. In the next section (4.3.2) this short duration, large amplitude event is interpreted as an azimuthally moving current tube, with a tailward current. There are however difficulties associated with this interpretation. For example.there is a reversal in $B_{z}(4)$, associated with the maximum of $B_{x}(4)$, but there is a time shift between the zero of $B_{z}(4)$ and the maximum of $B_{x}(4)$, which is not expected for a moving cylindrical current tube. These signatures could also be produced by multiple and tailward/eastward moving filamentary structures located at the front of the tailward fast flow.

It should be noted that these transient features are not only a minor small features but can be essential in the reconnection process, since it is during this time interval, that most accelerated electrons are observed. Imada et al. (2007) showed how the supra-thermal electrons appeared associated with another $B_{z}$ transient around 09:48:50 UT, which could well be explained by acceleration of bounced population at the front of the outward flow region as predicted by Hoshino et al. (1998) in PIC simulations. Large-amplitude (up to $50 \mathrm{mV} / \mathrm{m}$ ) solitary waves, identified as electron holes, were seen near the outer edge of the plasma sheet, within and on the edge of a density cavity, at distances on the order of a few ion inertial lengths from the center of the current sheet between 09:47 and 09:51 UT (Cattell et al., 2005). Characteristics of the electron holes, the association with electron beams and the density cavity and the location along the separatrices were consistent with reconnection simulations of Drake et al. (2003) with a guide field. In contrary to the large scale Hall$B_{y}$ observed from the current sheet crossings, this comparison rather suggest that electron holes may sometimes be an 
intrinsic feature of magnetotail reconnection and that effect of a guide field exists also in this event.

As can be seen in Fig. 9, there are significant contributions from oxygen during this interval. The heavy Oxygen possibly allowed us to observe ion-scale phenomena even with a quite large separation between the spacecraft. Effects of multi-component plasma in reconnection have been studied by simulations, which predicted different speeds for oxygen and hydrogen ions (Shay and Swisdak, 2004), which was also the case for the 1 October event (not shown). The $\mathrm{O}^{+}$in the reconnection region was suggested to experience a ballistic acceleration (Wygant et al., 2005) based on the observation of a large amplitude bipolar electric field $(60 \mathrm{mV} / \mathrm{m})$ observed by EFW directed normal to the current sheets for the same event.

In summary, this active substorm current sheet shows overall features quite consistent with reconnection. There is no clear guide field observed for this case, and the overall $B_{y}$ changes associated with $B_{x}$ changes were consistent with those due to Hall-effect in an X-line geometry. Yet, the event cannot be explained by a simple tailward retreat of a largescale X-line since several reversal of tailward to Earthward flow were observed. Furthermore, there are transient features with large amplitude in $B_{z}$ and $B_{y}$, indicating that such processes should be localized also in $Y$. Such three dimensional, transient structures can play a significant role in electron dynamics. That is, even if there is a background 2-D $\mathrm{X}$-line type configurations with time scale exceeding $10 \mathrm{~min}$, it contains essential processes taking place at least as localized transient phenomena, from several ion gyro scale down to electron scale.

\subsubsection{Event 2: Interpretation with CD model}

The overall good relationships between negative $B_{z}$ and tailward flows and positive $B_{z}$ and Earthward flow are interpreted in the CD model as being due to the balance between the amount of current reduction due to disruption and the ambient field as discussed in Sect. 2.3.

The large amplitude oscillations $(T \sim 100 \mathrm{~s})$ observed during Event 2 can correspond to an anti-symmetric kink mode (see, for instance, Daughton, 2002, for more details) which gives an odd parity perturbation for the current density $J_{y}$ (see Fig. 2), driven unstable by a large azimuthal current $J_{y}$, or to an anti-symmetric ballooning modes, also with an odd parity of the current density perturbation, driven unstable by the pressure gradient associated with this current. In the CD model the large amplitude fluctuations play an active role; they modulate $J_{y}$, which produces, via $\nabla \cdot \mathbf{J}=0$, localized field aligned current structures $\left(J_{x}\right)$. In other words the magnetic structure is 3-D; it results from the combined effects of a modulation of $J_{y}$, and the signatures of FAC. On the other hand an X-line, should produce planar sheets of Hall currents along the separatrices, and therefore have a large spatial extension along $Y$ (quasi-invariance by translation along $Y$ ). In the NENL model a bipolar signature on $B_{z}$ should be very rare, and correspond to a radially moving $\mathrm{X}$-line passing by the $\mathrm{S} / \mathrm{C}$ (see discussion above). It is therefore important to analyze the $B_{y}, B_{z}$ signatures on the various $\mathrm{S} / \mathrm{C}$.

During the 09:46-09:51 UT period we observe magnetic signatures that can be interpreted as signatures of localized FAC currents, in particular at 09:47:10, 09:47:45, and 09:47:40 UT.

From the signatures being different at different S/C locations, we infer that the transverse size of these structures is smaller than the distance between the S/C (about 1500$2000 \mathrm{~km}$ ), at least along $Z$ (see Fig. 7). In particular S/C 1, hardly detects the signature of the structure. S/C 1 being the northernmost S/C this lack of detection indicates that the structure has a small size, and is located well below S/C 1 . The signature of the structure involves a large positive excursion of $B_{y}$, on S/C 2 and S/C 4, with very similar temporal profiles. S/C 2 being to the west of S/C 4 the delay between the By signatures at S/C 2 and 4 suggests an azimuthal propagation of the structure, as mentioned in Section 3.3.2. The maximum of $B_{y}(2)$, red dashed line, is associated with a reversal in $B_{z}$, as expected for an azimuthally moving current tube. There is also a reversal in $B_{z}(4)$, associated with the maximum of $B_{x}(4)$, but there is a small time shift between the zero of $B_{z}(4)$ and the maximum of $B_{x}(4)$, which is not expected for a moving current tube. This time shift maybe due to a non null ambient $B_{z}$, which can be associated with the thickening of current sheet, as monitored by the decrease of $B_{x}(1)$, in average. Unlike $B_{y}(2)$ and $B_{y}(4), B_{y}(3)$ does not yield a large positive excursion. Yet $B_{z}(3)$ shows a clear reversal, from positive to negative, with an amplitude as large as for S/C 2 and S/C 4; hence S/C 3 is not farther from the structure than S/C 2 and S/C 4 . Given the lower location of S/C 3 we are led to conclude that S/C 3 crosses the cylindrical current structure near its center, thereby explaining why the signature on $B_{y}(3)$ is weak, while the signature on $B_{z}(3)$ is large. The large positive excursion of $B_{x}(3)$ observed at the same time as the reversal in $B_{z}(3)$, is consistent with this interpretation. Indeed a large axial field, pointing along the direction of the current, is found in the central region of most current tubes models (see, e.g., Slavin et al., 2003), including force-free models. This also explains why $B_{x}(3)>B_{x}(2)$ and $B_{x}(4) ; B_{x}$ should be larger at the $\mathrm{S} / \mathrm{C}$ which is the closest to the center of the current tube. Thus, apart from the delay mentioned above, the magnetic signatures at the various S/C are consistent with a cylindrical current structure carrying a tailward current, and moving eastward, with a small impact parameter for S/C 3, and passing successively below S/C 2 and S/C 4 . The cross section of the cylinder (in the $Y Z$ plane) is smaller than the distance, projected along $Z$, between S/C 3 and S/C 1 , and comparable to the distance between S/C 3 and S/C 4 (also along $Z$ ), namely a few $100 \mathrm{~km}$ along $Z$. The delay between the peaks on $B_{y}$ at S/C 2 and at $\mathrm{S} / \mathrm{C} 4$ is $\sim 5 \mathrm{~s}$, for a distance of about $1000 \mathrm{~km}$ (see Fig. 7), then the cylindrical current structure moves eastward 
at $\sim 200 \mathrm{~km} / \mathrm{s}$. This delay is comparable to the characteristic time of crossing of the structure. Hence the size of the tube is about $1000 \mathrm{~km}$, along $Y$.

Assuming a cylindrical tube with a radius of about $1000 \mathrm{~km}$, for $\delta B \approx 40 \mathrm{nT}$, we get $J_{x} \approx 60 \mathrm{nA} / \mathrm{m}^{2}$ (tailward), corresponding to a parallel drift between electrons and ions of $4000 \mathrm{~km} / \mathrm{s}$ (for $N \approx 0.1 / \mathrm{cm}^{3}$ ). Data from PEACE are consistent with the direction and the order of magnitude of the current given above (on S/C 3 we get $J_{x} \approx-110 \mathrm{nA} / \mathrm{m}^{2}$ ). Figure $16 \mathrm{~b}$ further illustrates the nature of the magnetic field structure at 09:47:45 UT. Spacecraft are again ordered by their positions along $Z_{\mathrm{GSM}}$. Using $B_{x}$ as a proxy for the distance with respect to the CS center, we find that the rotation of the vector $\mathbf{B}$, projected onto the $(Y Z)$ plane, is not consistent with a planar current sheet, quasi-invariant along $Y$. Indeed the magnetic field variations, shown in Fig. 16b, especially on S/C 2 and S/C 4, give evidence for rotations of the magnetic field in a plane perpendicular to $X$. As for Fig. 13b, the dashed circles have been built as tangent to the magnetic field vector at S/C 2, taken at three arbitrary times indicated by dashed arrows. The eastward motion of these centers confirms that a more or less cylindrical structure moves eastward. Magnetic signatures are consistent with a $J_{x}<0$ current filament (i.e. a tailward current structure moving eastward). The agreement is quite good, at least before 09:47:50 UT. After 09:47:50 UT the $B_{y}$ component, at S/C 3, becomes negative over a time scale longer than the duration of the structure, hence the quasi constant negative $B_{y}$, observed after 09:47:50 UT, is not considered as a signature of this structure. The rotation of the magnetic vector, together with the time lags between maxima on $B_{y}$ and $B_{z}$, confirm that we are crossing filamentary current structure and that the S/C cross (S/C 3), or pass by (S/C 2 and S/C 4) the current filament not only in the west- east direction (as for 7 September), but also in the north- south direction (because the CS is flapping).

\section{Conclusions}

The multi-point observations of Cluster S/C are particularly useful to unfold the nature of thin current sheets. For Event 1, a thin $\mathrm{CS}\left(H \sim 2000 \mathrm{~km} \sim 2 \rho_{i}\right)$ was found to be quiet, and to have a current density $J_{y}$ carried by ions. Conversely a slightly thinner CS $\left(H \sim 1000-2000 \mathrm{~km} \sim 1-2 \rho_{i}\right)$, crossed about $30 \mathrm{~min}$ later, was found to be active, with a mixture of fast earthward ion flow bursts $(300-600 \mathrm{~km} / \mathrm{s})$, together with somewhat slower quasi-steady flows $(200-400 \mathrm{~km} / \mathrm{s})$, and simultaneous large amplitude fluctuations $(\delta B / B \sim 1)$. During $\sim 20$ min (21:32-21:52 UT) the CS thickness remains small (though with large fluctuations); a large thickening only occurs at 21:52 UT. Thus an active CS can remain "metastable" for quite a long time. In this active CS the electron flow velocity $V_{x e}$ is, in average, close to the ion flow velocity $V_{x i}$, hence we can speak of a flow. Yet $V_{x e}$ fluc- tuates much more than $V_{x i}$. These fluctuations can be due to small scale field-aligned currents $\left(J_{x}\right)$, or to uncertainties in the determination of the electron velocity, in a situation where the ratio of the electron velocity to the thermal velocity is much smaller than unity. The estimated ion velocity $V_{y_{i}}$ is eastward, during the active period, 21:32-21:52 UT, but the average $V_{y_{e}}$ is also eastward, and larger in absolute value. Hence the differential drift between electrons and ions can carry the westward current. Furthermore low-frequency electromagnetic fluctuations are found to move in the same (eastward) direction as particles. During the active period fast earthward flows (300-600 km/s) are observed by S/C located in the CS, which suggests that the flow velocity maximizes at the CS center. The persistence of this earthward flow is difficult to explain; it implies a quasi-steady acceleration at or beyond Cluster, for about $20 \mathrm{~min}$. During the active phase of Event 1 no signature of the crossing of an $\mathrm{X}$-line is identified. Signatures of local dipolarizations are found (in particular near 21:36 and 21:52 UT) but their durations are shorter than the duration of the active period. An $\mathrm{X}$-line and/or a CD, located beyond Cluster, could account for the observed Earthward flow, if they can last $20 \mathrm{~min}$. In the later case, the motion of the dipolarization and the corresponding dissipation/diffusion of the current would have to occur beyond Cluster, and to be quite slow $(100 \mathrm{~km} / \mathrm{s}$ for $20 R_{E}$ ). The first large amplitude fluctuation and flow burst observed around 21:36 UT might correspond to the passage of this dipolarization front at Cluster. These large amplitude perturbations move eastward, which suggests that the reduction in the tail current is achieved via a series of eastward traveling partial dipolarizations/current disruptions. Keep in mind that Event 1 is a probably a pseudo-breakup developing only at high latitudes.

The second event is much more active than the first one. The observed flapping of the CS corresponds to an azimuthally propagating wave. A reversal in the proton flow velocity, from -1000 to $+1000 \mathrm{~km} / \mathrm{s}$, has been identified on protons measured by CODIF, during Event 2 (at 09:48 UT). Significant $\mathrm{O}^{+}$contribution in pressure, density but rather minor contribution in current was observed. The $\mathrm{O}^{+}$flow velocity also reverses, but the velocity jump is much smaller $(-100$ to $+100 \mathrm{~km} / \mathrm{s})$. This multi-component plasma effect could have affected the structure (scale-size) of the "thin" current sheet and the acceleration process of these particles. The overall flow reversals and associated changes in sign of $B_{z}$ and relationships between $B_{x}$ and $B_{y}$ suggest observation of $\mathrm{X}$ - line crossings and associated Hall-effect observations. In particular, the magnetic signatures from four spacecraft during the second tailward-to-Earthward flow reversal, between 09:47 UT and 09:51 UT, have been successfully interpreted in terms of Hall currents associated with an X- line that moves tailward around the S/C (see also Runov et al., 2003). Yet, we cannot explain the period by a simple tailward retreat of a large-scale X-line since several reversal of tailward to Earthward flow were observed. If CD is achieved 
via ballooning-like modes, periodic flow reversals are indeed expected, at least in the frame of the linear theory. Nonlinearly the flow direction should be determined by the sign of the averaged $B_{z}$, as discussed in the theory section.

For both events transient magnetic signatures (essentially in the $B_{y}, B_{z}$ plane) are observed, together with fast ion flows. These signatures have been used to test the two types of models. In a NENL scenario with large scale 2-D reconnection, the magnetic signatures in the S/C frame are interpreted as motions, along the $\mathrm{X}$ or $\mathrm{Z}$ direction, with respect to the $\mathrm{S} / \mathrm{C}$ of a quasi-steady $\mathrm{X}$-line. In the $\mathrm{CD}$ scenario the signatures in the $\mathrm{S} / \mathrm{C}$ frame are interpreted as signatures of azimuthally (along $Y$ ) moving current system associated with low frequency fluctuations of $J_{y}$ and the corresponding FAC system $\left(J_{x}\right)$. For Event 2 a very large magnetic signature occurs at 09:47:45 $\mathrm{UT}$, while $V_{x}\left(\mathrm{H}^{+}\right) \sim 0$. This occurs just before the reversal in $V_{x}\left(\mathrm{H}^{+}\right)$discussed above. It is tempting to interpret this magnetic signature as the crossing(s) of separatrices and/or X-line. However there is no simple way to interpret this signature by the radial/vertical motion of the $\mathrm{S} / \mathrm{C}$ with respect to a Hall structure. On the other hand its magnetic signature essentially corresponds to a structure of filamentary FAC $\left(J_{x}<0\right)$ moving eastward at $\sim 200 \mathrm{~km} / \mathrm{s}$, and passing by the various $\mathrm{S} / \mathrm{C}$, with different impact parameters. The transverse size of the structure is $\sim 1000 \mathrm{~km}$. Similar structures (though with less prominent signatures) are observed before and after. These filamentary structures are consistent with the eastward propagation of an azimuthal modulation $\left(K_{y}\right)$ associated with a current system $J_{y}, J_{x}$. They are not consistent with the azimuthal invariance which is expected if X-line(s) were structuring the plasma at or beyond the Cluster S/C. During Event 1 we also observe the signatures of filamentary FAC structures (see Fig. 13), associated with modulations of $J_{y}$. Thus, for both events, the structure of the magnetic fields is 3-D and corresponds to fluctuations of $J_{y}$, and to the associated FAC structures $\left(J_{x}\right)$. These 3-D structures move azimuthally. The 2-D NENL model fails at explaining the observed azimuthal structure of the currents. That is, even if there is a background 2-D X-line type configuration, with a time scale exceeding $10 \mathrm{~min}$, it can contain essential processes taking place at least as localized transient phenomena, from several ion gyro scales down to the electron scale in 3-D.

Evidence has been given for large amplitude "High Frequency" waves ( $F \geq$ few $\mathrm{Hz}$ ); the electric component is displayed in Fig. 12. Further studies are needed to characterize these waves and their possible effect on electron acceleration. The thickness of CS can be less than the distance between the $\mathrm{S} / \mathrm{C}$ which was $2000 \mathrm{~km}$ for the 2001 period considered here. With this relatively large inter-satellite distance the current density inside very thin CS could not be accurately estimated from the curlometer. At the time of the ISSI meeting smaller distances were not available. Current studies, based upon data from $2003(200 \mathrm{~km})$ and $2004(1000 \mathrm{~km})$ are underway or most recently completed (Nakamura et al., 2006b).
Acknowledgements. We would like to thank all members of the ISSI Substorm Onset Physics Team, who took part in the first meeting but could not make it to the second, for their valuable input: B. McPherron, H. Opgenoorth, G. Parks, P. Pritchett, T. Pulkkinen, M. Scholer, V. Sergeev, and T. Tanaka. All members of the Substorm Onset Physics Team are grateful to the International Space Science Institute in Bern, Switzerland, and its members for their hospitality, the great atmosphere, and their financial support. The participation of A. T. Y. Lui was supported by NSF Grant ATM0135667 to the Johns Hopkins University Applied Physics Laboratory. J. Birn also acknowledges NASA support from its Sun-Earth Connection Theory Program.

Topical Editor I. A. Daglis thanks V. Sergeev and another anonymous referee for their help in evaluating this paper.

\section{References}

Asano, Y., Mukai, T., Hoshino, M., Saito, Y., Hayakawa, H., and Nagai, T.: Current sheet structure around the near-Earth neutral line observed by Geotail, J. Geophys. Res., 109, A02212, doi:10.1029/2003JA010114, 2004.

Asano, Y., Nakamura, R., Baumjohann, W., Runov, A., Vörös, Z., Volwerk, M., Zhang, T. L., Balogh, A., Klecker, B., and Rème, H.: How typical are atypical current sheets?, Geophys. Res. Lett., 32, L03108, doi:10.1029/2004GL021834, 2005.

Baker, D. N., Pulkkinen, T. I., Angelopoulos, V., Baumjohann, W., and McPherron, R. L.: Neutral line model of substorms: Past results and present view, J. Geophys. Res., 101, 12 975-13 010, 1996.

Balogh, A., Carr, C. M., Acuña, M. H., Dunlop, M. W., Beek, T. J., Brown, P., Fornacon, K.-H., Georgescu, E., Glassmeier, K.-H., Harris, J., Musmann, G., Oddy, T., and Schwingenschuh, K.: The Cluster magnetic field investigation: Overview of in-flight perfomance and initial results, Ann. Geophys., 19, 1207-1217, 2001, http://www.ann-geophys.net/19/1207/2001/.

Baumjohann, W.: Modes of convection in the magnetotail, Phys. Plasmas, 9, 3665-3667, doi:10.1063/1.1499116, 2002.

Baumjohann, W., Paschmann, G., and Lühr, H.: Pressure balance between lobe and plasma sheet, Geophys. Res. Lett., 17, 45-48, 1990.

Baumjohann, W., Hesse, M., Kokubun, S., Mukai, T., Nagai, T., and Petrukovich, A. A.: Substorm dipolarization and recovery, J. Geophys. Res., 104, 24 995-25 000, 1999.

Bhattacharjee, A., Ma, Z. W., and Wang, X.: Ballooning instability of a thin current sheet in the high-Lundquist number magnetotail, Geophys. Res. Lett., 25, 861-864, 1998a.

Bhattacharjee, A., Ma, Z. W., and Wang, X.: Dynamics of thin current sheets and their disruption by ballooning instabilities: A mechanism for magnetospheric substorms, Phys. Plasmas, 5, 2001-2009, 1998b.

Birn, J. and Hesse, M.: The substorm current wedge and fieldaligned currents in MHD simulations of magnetotail reconnection, J. Geophys. Res., 96, 1611-1618, 1991.

Birn, J. and Hesse, M.: Details of current disruption and diversion in simulations of magnetotail dynamics, J. Geophys. Res., 101, 15 345-15 358, 1996.

Birn, J. and Schindler, K.: Thin current sheets in the magnetotail and the loss of equilibrium, J. Geophys. Res., 107, 1117, 
doi:10.1029/2001JA0291, 2002.

Birn, J., Hesse, M., and Schindler, K.: Formation of thin current sheets in space plasmas, J. Geophys. Res., 103, 6843-6852, 1998.

Birn, J., Hesse, M., Haerendel, G., Baumjohann, W., and Shiokawa, K.: Flow braking and the substorm current wedge, J. Geophys. Res., 104, 19 895-19904, 1999.

Brackbill, J. U., Forslund, D. W., Quest, K. B., and Winske, D.: Nonlinear evolution of the lower-hybrid drift instability, Phys. Fluids, 27, 2682-2693, 1984.

Büchner, J.: Transition from small scale turbulence to large scale coherent reconnection, in: Physics of Space Plasma, edited by: Chang, T., Crew, G. B., and Jasperse, J. R., pp. 382-388, Scientific Publishers, Inc., Cambridge, Massachusetts, USA, 1998.

Büchner, J. and Kuska, J.-P.: Sausage mode instability of thin current sheets as a cause of magnetospheric substorms, Ann. Geophys., 17, 604-612, 1999, http://www.ann-geophys.net/17/604/1999/.

Cattell, C., Dombeck, J., Wygant, J., Drake, J. F., Swisdak, M., Goldstein, M. L., Keith, W., Fazakerley, A., André, M., Lucek, E., and Balogh, A.: Cluster observations of electron holes in association with magnetotail reconnection and comparison to simulations, J. Geophys. Res., 110, A01211, doi:10.1029/2004JA010519, 2005.

Chanteur, G.: Spatial interpolation for four spacecraft: Theory, in: Analysis Methods for Multi-Spacecraft Data, edited by: Paschmann, G. and Daly, P., pp. 349-369, ESA, Noordwijk, 1998.

Chen, L.: Theory of plasma transport induced by low-frequency hydromagnetic waves, J. Geophys. Res., 104, 2421-2428, 1999.

Cheng, C. Z. and Lui, A. T. Y.: Kinetic ballooning instability for substorm onset and current disruption observed by AMPTE/CCE, Geophys. Res. Lett., 25, 4091-4094, 1998.

Daughton, W.: The unstable eigenmodes of a neutral sheet, Phys. Plasmas, 6, 1329-1343, 1999a.

Daughton, W.: Two-fluid theory of the drift kink instability, J. Geophys. Res., 104, 28 701-28 707, 1999b.

Daughton, W.: Nonlinear dynamics of thin current sheets, Phys. Plasmas, 9, 3668-3678, 2002.

Drake, J. F., Swisdak, M., Cattell, C., Shay, M. A., Rogers, B. N., and Zeiler, A.: Formation of Electron Holes and Particle Energization During Magnetic Reconnection, Science, 299, 873-877, 2003.

Gustafsson, G., André, M., Carozzi, T., Eriksson, A. I., Fälthammar, C. G., Grard, R., Holmgren, G., Holtet, J. A., Ivchenko, N., Khotyaintsev, T. K. Y., Klimov, S., Laakso, H., Lindqvist, P. A., Lybekk, B., Marklund, G., Mozer, F., Mursula, K., Pedersen, P., Popielawska, B., Savin, S., Stasiewicz, K., Tanskanen, P., Vaivads, A., and Wahlund, J. E.: First results of electric field and density observations by CLUSTER EFW based on initial months of operation, Ann. Geophys., 19, 1219-1240, 2001, http://www.ann-geophys.net/19/1219/2001/.

Harris, E. G.: On a plasma sheet separating regions of oppositely directed magnetic field, Nuovo Cimento, 23, 115-121, 1962.

Hesse, M. and Birn, J.: On dipolarization and its relation to the substorm current wedge, J. Geophys. Res., 96, 19417-19426, 1991.

Hesse, M. and Birn, J.: Near- and mid-tail current flow during substorms: Small- and large-scale aspects of current disruption, in:
Magnetospheric Current Systems, Geophys. Monogr. 118, edited by: Ohtani, S., Fujii, R., Hesse, M., and Lysak, R., p. 295, AGU, Washington, D.C., 2000.

Hesse, M. and Kivelson, M. G.: The Formation and Structure of Flux Ropes in the Magnetotail, in: New Perspectives on the Earth's Magnetotail, edited by: Nishida, A., Baker, D. N., and Cowley, S. W. H., p. 139, 1998.

Hesse, M. and Winske, D.: Electron dissipation in collisionless magnetic reconnection, J. Geophys. Res., 103, 26479-26486, 1998.

Hesse, M., Winske, D., and Kuznetsova, M. M.: Hybrid modeling of collisionless reconnection in two-dimensional current sheets: Simulations, J. Geophys. Res., 100, 21 815-21 826, 1995.

Hesse, M., Winske, D., Kuznetsova, M., Birn, J., and Schindler, K.: Hybrid modeling of the formation of thin current sheets in magnetotail configurations, J. Geomag. Geoelec., 48, 749-763, 1996.

Hesse, M., Schindler, K., Birn, J., and Kuznetsova, M.: The diffusion region in collisionless magnetic reconnection, Phys. Plasmas, 6, 1781-1795, 1999.

Hesse, M., Birn, J., and Kuznetsova, M.: Collisionless magnetic reconnection: Electron processes and transport modeling, J. Geophys. Res., 106, 3721-3736, 2001a.

Hesse, M., Kuznetsova, M., and Birn, J.: Particle-in-cell simulations of three-dimensional collisionless magnetic reconnection, J. Geophys. Res., 106, 29 831-29 842, 2001 b.

Hewett, D. W., Frances, G. E., and Max, C. E.: New regimes of magnetic reconnection in collisionless plasmas, Phys. Rev. Lett., 61, 893-896, 1988.

Horiuchi, R. and Sato, T.: Three-dimensional particle simulation of plasma instabilities and collisionless reconnection in a current sheet, Phys. Plasmas, 6, 4565-4574, 1999.

Hoshino, M., T.Mukai, Yamamoto, T., and Kokubun, S.: Ion dynamics in magnetic reconnection: Comparison between numerical simulation and Geotail observations, J. Geophys. Res., 103, 4509-4530, 1998.

Hoshino, M., Mukai, T., Terasawa, T., and Shinohara, I.: Superthermal electron acceleration in magnetic reconnection, J. Geophys. Res., 106, 25,972-25,997, 2001.

Huba, J. D., Gladd, N. T., and Papadopoulos, K.: Lower-hybriddrift wave turbulence in the distant magnetotail, J. Geophys. Res., 83, 5217-5226, 1978.

Hurricane, O. A., Pellat, R., and Coroniti, F. V.: A new approach to low-frequency "MHD-like" waves in magnetospheric plasmas, J. Geophys. Res., 100, 19421-19427, 1995.

Hurricane, O. A., Fong, B. H., and Cowley, S. C.: Nonlinear magnetohydrodynamic detonation, Part I, Phys. Plasmas, 4, 35653580, 1997.

Imada, S., Nakamura, R., Daly, P. W., Hoshino, M., Baumjohann, W., Mühlbachler, S., Balogh, A., and Rème, H.: Energetic electron acceleration in the downstream reconnection outflow region, J. Geophys. Res., 112, A03202, doi:10.1029/2006JA011847, 2007.

Khurana, K. K., Kivelson, M. G., Frank, L. A., and Paterson, W. R.: Observations of magnetic flux ropes and associated currents in Earth's magnetotail with the Galileo spacecraft, Geophys. Res. Lett, 22, 2087-2090, 1995.

Kistler, L., Mouikis, C., Möbius, E., Klecker, B., Sauvaud, J.-A., Rème, H., Korth, A., Marocci, M. F., Lundin, R., Parks, G. K., 
and Balogh, A.: Contribution of nonadiabatic ions to the crosstail current and $\mathrm{O}^{+}$dominated thin current sheet, J. Geophys. Res., 110, A06213, doi:10.1029/2004JA010653, 2005.

Kivelson, M. G., McPherron, R. L., Thompson, S., Khurana, K. K., Weygand, J. M., and Balogh, A.: The response of the near Earth magnetotail to substorm activity, Adv. Space Res., 36, 18181824, doi:10.1016/j.asr.2004.03.024, 2005.

Lapenta, G. and Brackbill, J. U.: A kinetic theory for the drift-kink instability, J. Geophys. Res., 102, 27 099-27 108, 1997.

Lapenta, G. and Brackbill, J. U.: 3D reconnection due to oblique modes: a simulation of Harris current sheets, Nonlin. Processes Geophys., 7, 151-158, 2000,

http://www.nonlin-processes-geophys.net/7/151/2000/.

Lapenta, G. and Knoll, D. A.: Reconnection in the Solar Corona: Role of the Kelvin-Helmholtz Instability, Solar Phys., 214, 107129, 2003.

Le Contel, O., Pellat, R., and Roux, A.: Self-consistent quasi-static radial transport during the substorm growth phase, J. Geophys. Res., 105, 12 929-12 944, 2000a.

Le Contel, O., Pellat, R., and Roux, A.: Self-consistent quasi-static parallel electric field associated with substorm growth phase, J. Geophys. Res., 105, 12945-12 954, 2000b.

Le Contel, O., Perraut, S., Roux, A., and Pellat, R.: Plasma transport during substorm growth phase and relation to breakup, Space Sci. Rev., 95, 415-426, 2001a.

Le Contel, O., Roux, A., Perraut, S., Pellat, R., Holter, O., Pedersen, A., and Korth, A.: Possible control of the plasma transport in the near-Earth plasma sheet via current driven Alfvèn waves, J. Geophys. Res., 106, 10 817-10 827, 2001b.

Le Contel, O., Roux, A., Perraut, S., et al.: Role of the Parallel Current Instability during Substorms: Theory and Observations, in: Proc. 6th Int. Conf. on Substorm (ICS-6), edited by: Winglee, R. M., pp. 326-334, University of Washigton, Seatle, USA, 2002.

Lui, A. T. Y.: A synthesis of magnetospheric substorm models, J. Geophys. Res., 96, 1849-1856, 1991.

Lui, A. T. Y.: Current disruption in the Earth's magnetosphere: Observations and models, J. Geophys. Res., 101, 13067-13088, 1996.

Lui, A. T. Y.: A multiscale model for substorms, Space Sci. Rev., 95, 325-345, 2001.

Lui, A. T. Y., Yoon, P. H., and Chang, C.-L.: Quasi-linear analysis of ion Weibel instability, J. Geophys. Res., 98, 153-163, 1993.

McPherron, R. L., Russell, C. T., and Aubry, M. A.: Satellite studies of magnetospheric substorms on August 15, 1968, 9, Phenomenological model for substorms, J. Geophys. Res., 78, 31313149, 1973.

Mitchell, D. G., Williams, D. J., Huang, C. Y., Frank, L. A., and Russel, C. T.: Current carriers in the near-Earth cross-tail current sheet during substorm growth phase, Geophys. Res. Lett., 17, 583-586, 1990.

Nakamura, R., Baumjohann, W., Runov, A., Volwerk, M., Zhang, T. L., Klecker, B., Bogdanova, Y., Roux, A., Balogh, A., Rème, H., Sauvaud, J.-A., and Frey, H. U.: Fast flow during current sheet thinning, Geophys. Res. Lett., 29, 2140, doi:10.1029/2002GL016200, 2002.

Nakamura, R., Amm, O., Laakso, H., Draper, N., Lester, M., Grocott, A., Klecker, B., McCrea, I. W., Balogh, A., Rème, H., and André, M.: Localized fast flow disturbance observed in the plasma sheet and in the ionosphere, Ann. Geophys., 23, 553566,2005 ,

http://www.ann-geophys.net/23/553/2005/.

Nakamura, R., Baumjohann, W., Asano, Y., Runov, A., Balogh, A., Owen, C. J., Fazakerley, A. N., Fujimoto, M., Klecker, B., and Rème, H.: Dynamics of thin current sheets associated with magnetotail reconnection, J. Geophys. Res., 111, A11206, doi:10.1029/2006JA011706, 2006a.

Nakamura, R., Baumjohann, W., Runov, A., and Asano, Y.: Thin current sheets in the magnetotail observed by Cluster, Space Sci. Rev., 122, 29-38, 2006b.

Nishikawa, K.-I.: Particle entry into the magnetosphere with a southward interplanetary magnetic field studied by a threedimensional electromagnetic particle code, J. Geophys. Res., 102, 17 631-17 642, 1997.

Ohtani, S., Kokubun, S., and Russell, C. T.: Radial expansion of the tail current disruption during substorms: A new approach to the substorm onset region, J. Geophys. Res., 97, 3129-3136, 1992.

Ozaki, M., Sato, T., Horiuchi, R., and the Complexity Simulation Group: Electromagnetic instability and anomalous resistivity in a magnetic neutral sheet, Phys. Plasmas, 3, 2265-2274, 1996.

Pellat, R., Le Contel, O., Roux, A., Perraut, S., Hurricane, O., Coroniti, F. V., and Luciani, J.-F.: Un nouveau mecanisme pour les sous-orages magnetospheriques, C. R. Acad. Sci. Paris, t.1, Serie IV, 1-6, 2000.

Perraut, S., Le Contel, O., Roux, A., and Pedersen, A.: Currentdriven electromagnetic ion cyclotron instability at substorm onset, J. Geophys. Res., 105, 21 097-21 107, 2000.

Pritchett, P. L.: Effect of electron dynamics on collisionless reconnection in two-dimensional magnetotail equilibria, J. Geophys. Res., 99, 5935-5941, 1994.

Pritchett, P. L.: Geospace Environment Modeling magnetic reconnection challenge: Simulations with a full particle electromagnetic code, J. Geophys. Res., 106, 3783-3798, 2001.

Pritchett, P. L. and Coroniti, F. V.: Convection and the formation of thin currents sheets in the near-Earth plasma sheet, Geophys. Res. Lett., 21, 1587-1590, 1994.

Pritchett, P. L. and Coroniti, F. V.: The role of drift kink mode in destabilizing thin current sheets, J. Geomag. Geoelec., 48, 833844, 1996.

Pritchett, P. L., Coroniti, F. V., and Decyk, V. K.: Three-dimensional stability of thin quasi-neutral current sheets, J. Geophys. Res., 101, 27 413-27 430, 1996.

Pu, Z. Y., Korth, A., Chen, Z. X., Friedel, R. H. W., Zong, Q. G., Wang, X. M., Hong, M. H., Fu, S. Y., Liu, Z. X., and Pulkkinen, T. I.: MHD drift ballooning instability near the inner edge of the near-Earth plasma sheet and its application to substorm onset, J. Geophys. Res., 102, 14 397-14 406, 1997.

Rème, H., Aostin, C., Bosqued, J. M., Dandouras, I., Lavraud, B., Sauvaud, J.-A., Barthe, A., Bouyssou, J., Camus, T., Coeur-Joly, O., et al.: First multispacecraft ion measurements in and near the Earth's magnetosphere with the identical Cluster ion spectrometry (CIS) experiment, Ann. Geophys, 19, 1303-1354, 2001.

Rogers, B. N., Drake, J. F., and Shay, M. A.: The onset of turbulence in collisionless magnetic reconnection, Geophys. Res. Lett., 27, 3157-3160, 2000.

Roux, A., Perraut, S., Robert, P., Morane, A., Pedersen, A., Korth, A., Kremser, G., Aparicio, B., Rodgers, D., and Pellinen, R.: Plasma sheet instability related to the westward traveling surge, 
J. Geophys. Res., 96, 17 697-17 714, 1991.

Runov, A., Nakamura, R., Baumjohann, W., Treumann, R. A., Zhang, T. L., Volwerk, M., Vörös, Z., Balogh, A., Glassmeier, K.-H., Klecker, B., Rème, H., and Kistler, L.: Current sheet structure near magnetic X-line observed by Cluster, Geophys. Res. Lett., 30, 1579, doi:10.1029/2002GL016730, 2003.

Runov, A., Sergeev, V. A., Nakamura, R., Baumjohann, W., Zhang, T. L., Asano, Y., Volwerk, M., Vörös, Z., Balogh, A., and Rème, H.: Reconstruction of the magnetotail current sheet structure using multi-point Cluster measurements, Planet. Space Sci., 53, 237-243, 2005.

Runov, A., Sergeev, V. A., Nakamura, R., Baumjohann, W., Apatenkov, S., Asano, Y., Takada, T., Volwerk, M., Vörös, Z., Zhang, T. L., Sauvaud, J.-A., Rème, H., and Balogh, A.: Local structure of the magnetotail current sheet: 2001 Cluster observations, Ann. Geophys, 24, 247-262, 2006.

Schindler, K. and Birn, J.: On the cause of thin current sheets in the near-Earth magnetotail and their possible significance for magnetospheric substorms, J. Geophys. Res., 98, 15 477-15 485, 1993.

Schindler, K. and Birn, J.: Models of two-dimensional embedded thin current sheets from Vlasov theory, J. Geophys. Res., 107, 1193, doi:10.1029/2001JA0304, 2002.

Scholer, M. and Otto, A.: Magnetotail reconnection: Current diversion and field-aligned currents, Geophys. Res. Lett., 18, 733736, 1991.

Sergeev, V., Runov, A., Baumjohann, W., Nakamura, R., Zhang, T. L., Volwerk, M., Balogh, A., Rème, H., Sauvaud, J.-A., André, M., and Klecker, B.: Current sheet flapping motion and structure observed by Cluster, Geophys. Res. Lett., 30, 1327, doi:10.1029/ 2002GL016500, 2003.

Sergeev, V., Runov, A., Baumjohann, W., Nakamura, R., Zhang, T. L., Balogh, A., Louarn, P., Sauvaud, J.-A., and Rème, H.: Orientation and propagation of current sheet oscillations, Geophys. Res. Lett., 31, L05807, doi:10.1029/2003GL019346, 2004.

Sergeev, V. A., Kubyshkina, M. V., Baumjohann, W., Nakamura, R., Amm, O., Pulkkinen, T., Angelopoulos, V., Mende, S. B., Klecker, B., Nagai, T., Sauvaud, J.-A., Slavin, J. A., and Thomsen, M. F.: Transition from substorm growth to substorm expansion phase as observed with a radial configuration of ISTP and Cluster spacecraft, Ann. Geophys., 23, 2183-2198, 2005, http://www.ann-geophys.net/23/2183/2005/.

Sergeev, V. A., Sormakov, D. A., Apatenkov, S. V., Baumjohann, W., Nakamura, R., Runov, A., Mukai, T., and Nagai, T.: Survey of large-amplitude flapping motions in the midtail current sheet, Ann. Geophys., 24, 2015-2024, 2006, http://www.ann-geophys.net/24/2015/2006/.

Shay, M. A. and Swisdak, M.: Three-Species Collisionless Reconnection: Effect of O+ on Magnetotail Reconnection, Phys. Rev. Lett., 93, 175 001, doi:10.1103/PhysRevLett.93.175001, 2004.

Shiokawa, K., Baumjohann, W., and Haerendel, G.: Breaking of high-speed flows in the near-Earth tail, Geophys. Res. Lett., 24, 1179-1182, 1997.
Slavin, J. A., Lepping, R. P., Gjerloev, J., Fairfield, D. H., Acuna, M. H., Goldstein, M. L., Balogh, A., Dunlop, M., Kivelson, M. G., Khurana, K., Fazakerley, A., Owen, C. J., Rème, H., and Bosqued, J. M.: Cluster measurements of electric current density within a flux rope in the plasma sheet, Geophys. Res. Lett., 30, 1362, doi:10.1029/2003GL016411, 2003.

Tanaka, M.: Macro-particle simulations of collisionless magnetic reconnection, Phys. Plasmas, 2, 2920-2930, 1995a.

Tanaka, M.: The macro-EM particle simulation method and a study of collisionless magnetic reconnection, Comp. Phys. Comm., 87, 117-138, 1995 b.

Thompson, S. M., Kivelson, M. G., El-Alaoui, M., Balogh, A., Rème, H., and Kistler, L. M.: Bifurcated current sheets: Statistics from Cluster magnetometer measurements, J. Geophys. Res., 111, A03212, doi:10.1029/2005JA011009, 1993.

Treumann, R. A., Jaroschek, C. H., Nakamura, R., Runov, A., and Scholer, M.: The role of the Hall effect in collisionless magnetic reconnection, Adv. Space. Res., 38, 101-111, 2006.

Wilber, M., Lee, E., Parks, G. K., Meziane, K., Carlson, C. W., McFadden, J. P., Rème, H., Dandouras, I., Sauvaud, J.-A., Bosqued, J.-M., Kistler, L., Möbius, E., McCarthy, M., Korth, A., Klecker, B., Bavassano-Cattaneo, M.-B., Lundin, R., and Lucek, E.: Cluster observations of velocity space-restricted ion distributions near the plasma sheet, Geophys. Res. Lett., 31, L24802, doi:10.1029/2004GL020265, 2004.

Wygant, J. R., Cattell, C. A., Lysak, R., Song, Y., Dombeck, J., McFadden, J., Mozer, F. S., Carlson, C. W., Parks, G., Lucek, E. A., Balogh, A., Andre, M., Rème, H., Hesse, M., and Mouikis, C.: Cluster observations of an intense normal component of the electric field at a thin reconnecting current sheet in the tail and its role in the shock-like acceleration of the ion fluid into the separatrix region, J. Geophys. Res., 110, A09206, doi:10.1029/2004JA010708, 2005.

Yoon, P. H. and Lui, A. T. Y.: On the drift-sausage mode in onedimensional current sheet, J. Geophys. Res., 106, 1939-1947, 2001.

Yoon, P. H., Drake, J. F., and Lui, A. T. Y.: Theory and simulation of Kelvin-Helmholtz instability in the geomagnetic tail, J. Geophys. Res., 101, 27 327-27 339, 1996.

Zeiler, A., Biskamp, D., Drake, J. F., Rogers, B. N., Shay, M. A., and Scholer, M.: Three-dimensional particle simulations of collisionless magnetic reconnection, J. Geophys. Res., 107, 1230, doi:10.1029/2001JA000287, 2002.

Zhang, T. L., Baumjohann, W., Nakamura, R., Balogh, A., and Glassmeier, K.-H.: A wavy twisted neutral sheet observed by Cluster, Geophys. Res. Lett., 29, 1899, doi:10.1029/2002GL015544, 2002.

Zhu, Z. and Winglee, R. M.: Tearing instability, flux ropes, and the kinetic current sheet kink instability in the Earth's magnetotail: A three-dimensional perspective from particle simulations, J. Geophys. Res., 101, 4885-4898, 1996. 